Brit. J. industr. Med., 1956, 13, 9.

\title{
FURTHER STUDIES OF THE DUST IN LUNGS OF COAL-MINERS
}

\author{
BY \\ E. J. KING, B. A. MAGUIRE, and G. NAGELSCHMIDT \\ From the Postgraduate Medical School of London and the Safety in Mines Research Establishment, Sheffield
}

(RECEIVED FOR PUblication AUgUST 23, 1955)

In a previous communication (King and Nagelschmidt, 1945), analytical data were given for the amount and composition of the dust found in the lungs of 54 coalworkers from South Wales. Coal, total silica, quartz, mica, and kaolin were determined as the percentages of dried lung tissue, and the results were related to type of work and degree of pathology. The main findings were the following :

(1) The kind of occupation was clearly revealed in the composition of the dust from the lungs. For instance, its coal percentage varied from 25 for rock-workers* to 95 for trimmers and screenworkers.

(2) The average dust composition in the lungs of 27 anthracite workers was very similar to the composition of the airborne dusts at the coal face, and thus there was no evidence of any mineral change in the lung, as far as the ratio of coal to non-coal or the relative proportions of mica, quartz, and kaolin were concerned.

(3) In the pathological classification three grades were distinguished : reticulation, mixed nodulation, and confluent fibrosis. In addition, three rockworkers were classed as a silicotic nodulation group. The concentrations of coal, quartz, and total silica varied considerably within each pathological group, but increased only very slightly from one group to the next, except for the silicotic nodulation group; in the more homogeneous group of the 27 anthracite colliers the increases in lung dust components with increasing grade of pathology were not significant at the $95 \%$ significance level.

In the discussion of the results it was stated that the silicotic nodulation was due to quartz, but that it could not be decided on the basis of the data whether reticulation was also a quartz effect, modified by the large amounts of coal and other \footnotetext{
* The reference to rock-workers throughout this paper means
rock-workers in coal-mines.
}

siliceous minerals, or whether it was a type of lung change due to the presence of large amounts of inert foreign material which could have been produced by equivalent amounts of pure coal without the associated siliceous components found in the airborne dusts. Information from lung residue data may not be able to solve this problem, but the dust in the lungs and the associated pathological changes are material evidence which cannot be neglected.

In order to gain further information more lungs have been analysed. In particular, attempts have been made to secure material from the earlier stages of the disease and from the whole range of coal rank found in South Wales. These attempts have only been partly successful, as will be shown later. The present report deals with the analyses of 71 further lungs of coalworkers from South Wales, and of 15 silicotic lungs of tin-miners and granite-workers from Cornwall, which are included for comparison. The 86 new lungs will in the following be called the "new series" in contrast to the 54 lungs described previously, which will be referred to as the "old series". Data on lungs of three men without known dust exposure have also been obtained.

It was originally intended to pool the information from the coalworkers of the old and new series in the hope that the indicative but not statistically significant results from the old series would become significant for the whole group. Such pooling could, however, not be done, as will be described later, because whole lungs were analysed in the new series as against selected fibrosed parts of lungs in the old one. The present results are therefore again in the nature of a progress report.

Ideally, one would want to measure dust exposures for groups of men and radiograph them periodically. After death, one would examine and analyse the lungs and could then relate radiographs, 
pathology, and analyses of dust in the lungs to the dust exposure. This is at present impossible, and the best that can be done is to relate the pathological data to the dust in the lungs and to such industrial histories and radiographs as can be obtained. As the taking of dust records improves and proceeds in British coal-mines, it will be possible, in the future, to have better exposure records for lungs that come to necropsy and analysis. The purpose of the present work is thus to see what relation, if any, there is between the amount of total dust or of any of its components and the type of pathological change found in the lungs.

\section{Source of Material}

Professor J. Gough has provided the following note on the source of the lungs and the criteria used in selecting these lungs :

All the necropsies were carried out at the Pathology Department of the Welsh National School of Medicine so that the lungs were immediately available for preparation. This was an advantage as the lungs could be dried without the addition of any fixative or other fluid. The placing of lungs in fixative has a disadvantage in that a considerable amount of dust may be washed out. The necropsies were on men who were known to have had pneumoconiosis, on those in whom it was alleged or suspected that pneumoconiosis was present although this had not been diagnosed during life, and also on miners dying in general hospitals from causes other than pneumoconiosis. The aim was to get an equal number of lungs from various grades of pneumoconiosis. A sufficient number of cases of massive fibrosis were quickly obtained, but the cases of simple pneumoconiosis were obtained over a much longer period. (Further details on the selection of lungs are given in the discussion.) The right lung was used for chemical analysis except for small portions reserved for histology and bacteriology. The hilar glands and main bronchi were excised and discarded and the lung tissue was then cut and placed in pyrex jars, dried at approximately $105^{\circ} \mathrm{C}$., and weighed. The left lung was used for preparing large sections.

There is one important difference in technique between the old and the new series. In the old series representative parts of lung tissue, usually amounting to about $200 \mathrm{~g}$. wet weight, were selected, dried, and analysed. It was therefore only possible to give dust concentrations (per cent. dry lung), but not the absolute amounts per lung. In the new series the whole of both lungs was available. The left lung in every case was used for pathological examination and for cutting large sections by the technique of Gough and Wentworth (1949). A representative sample of the whole right lung was used for analysis, and concentrations and absolute amounts of the total dust and of the different dust constituents were determined.

Badham and Taylor (1938) have discussed the problem of lung sampling fully; they cut the lungs in an axillary direction and used one half of the left and one half of the right lobes for analysis. But the cutting of the lungs either fixed or unfixed inevitably leads to the loss of some dust. In our opinion the analysis of the right (or left) lung is preferable, unless there are gross disparities between the two lungs. Such lungs were not included in the series.

\section{Classification}

In order to retain conformity with the old series the same pathological classification was used as before, but one earlier grade, slight reticulation, was added.

We therefore have the following five pathological grades : (1) slight reticulation, (2) reticulation, (3) mixed nodulation, (4) confluent fibrosis, and (5) silicotic nodulation.

Pictures of representative large sections from lungs in each of the categories are reproduced in Figs. 1 to 5. (We are indebted to Professor J. Gough for these illustrations.)

The radiological classification used was that of the Pneumoconiosis Research Unit described by Cochrane, Davies, and Fletcher (1951), which distinguishes three grades of simple pneumoconiosis and four grades of progressive massive fibrosis (P.M.F.). Its relation to the pathological grades is described on p. 16.

The occupational groups are also the same as in the old series, except that no separate groups of anthracite- and steam-coal colliers could be made, as most of the colliers had worked in steam-coal mines. A further group, "unclassifiable underground", had to be added which reflects the failure to obtain adequate working histories. All the groups are from South Wales except the last group of tin- and granite-workers from Cornwall, where

TABLE 1

NUMBER OF LUNGS IN NEW SERIES ACCORDING TO OCCUPATION AND PATHOLOGICAL GRADING

\begin{tabular}{|c|c|c|c|c|c|c|c|c|c|}
\hline \multirow{2}{*}{\multicolumn{4}{|c|}{ Occupation }} & \multicolumn{5}{|c|}{ Pathological Grade } & \multirow{2}{*}{ Total } \\
\hline & & & & $\frac{1}{1}$ & 2 & 3 & $\frac{4}{4}$ & $\frac{5}{-}$ & \\
\hline $\begin{array}{l}\text { Rock-work } \\
\text { Firemen-sh } \\
\text { Hauliers, ri } \\
\text { Colliers } \\
\text { Workers on } \\
\text { Not classif } \\
\text { Tin-miners }\end{array}$ & $\begin{array}{l}\text { ers } \\
\text { otsmen } \\
\text { ders, etc. } \\
\text { surface s } \\
\text { able } \\
\text { and grani }\end{array}$ & $\begin{array}{l}\ddot{ } \\
\ddot{y} \\
\ddot{\text { s }} \\
\dot{\text { rikers }}\end{array}$ & $\begin{array}{l}\cdots \\
\cdots \\
\cdots \\
\cdots\end{array}$ & $\begin{array}{l}1 \\
4 \\
1 \\
2 \\
3 \\
1\end{array}$ & $\begin{array}{l}1 \\
3 \\
8 \\
1 \\
7 \\
-\end{array}$ & $\begin{array}{l}\frac{3}{2} \\
\frac{9}{6} \\
-\end{array}$ & $\begin{array}{r}4 \\
\frac{3}{10} \\
\frac{2}{2}\end{array}$ & $\begin{array}{l}\overline{1} \\
\overline{1} \\
\overline{14}\end{array}$ & $\begin{array}{r}9 \\
3 \\
10 \\
28 \\
3 \\
18 \\
15\end{array}$ \\
\hline Total & . & .. & . & 12 & 20 & 20 & 19 & 15 & 86 \\
\hline
\end{tabular}




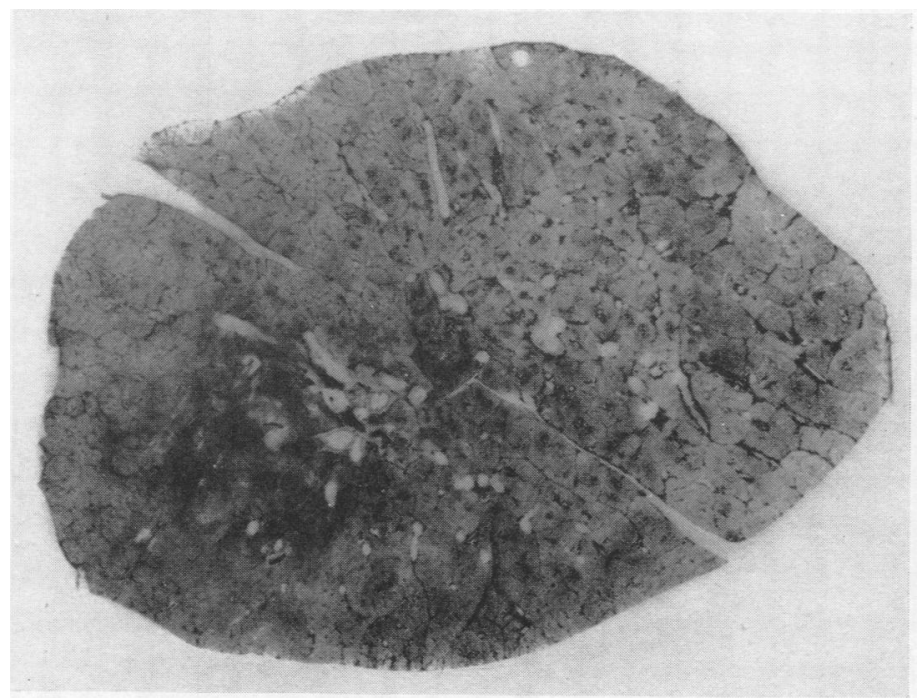

Fig. 1

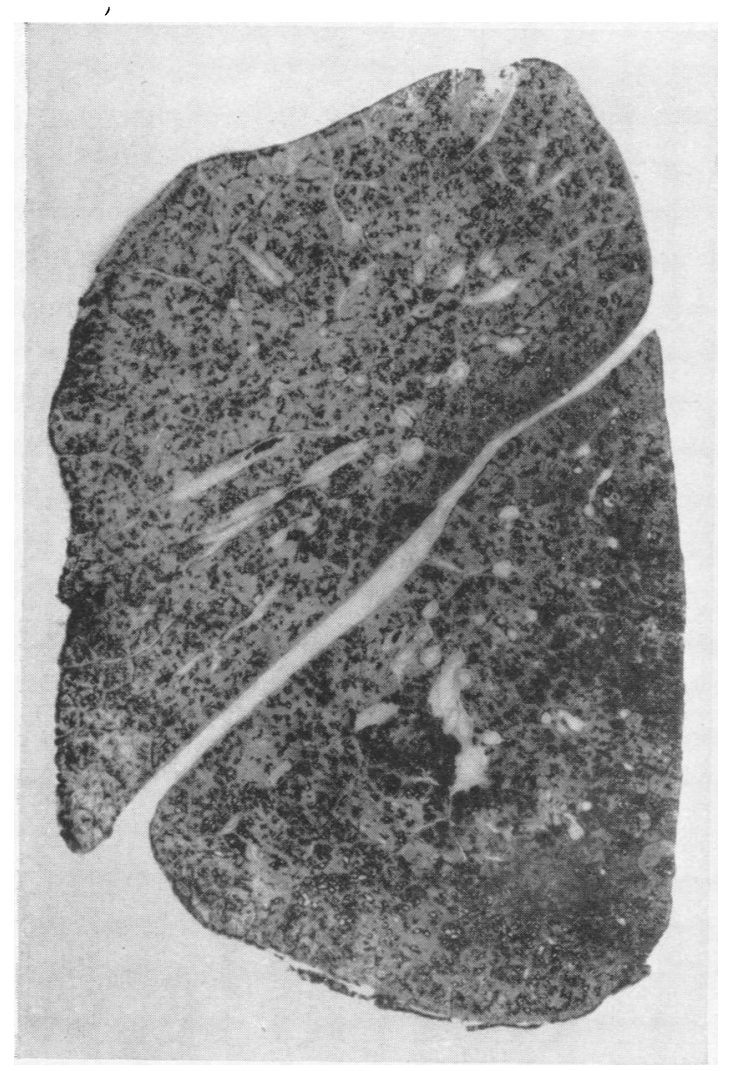

FIG. 2
FIG. 1.-Slight reticulation.

FIG. 2.-Reticulation.

FIG. 3.-Mixed nodulation.

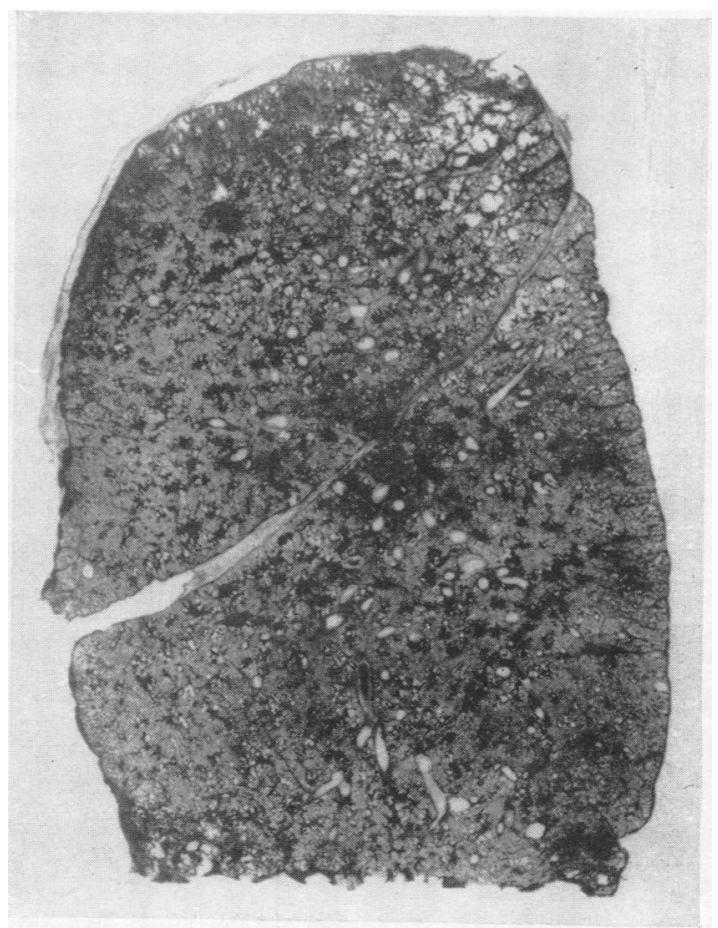

Fig. 3 
Fig. 4.-Confluent fibrosis.

FIG. 5.-Silicotic nodulation.
Fig. 5.

FIG. 4.

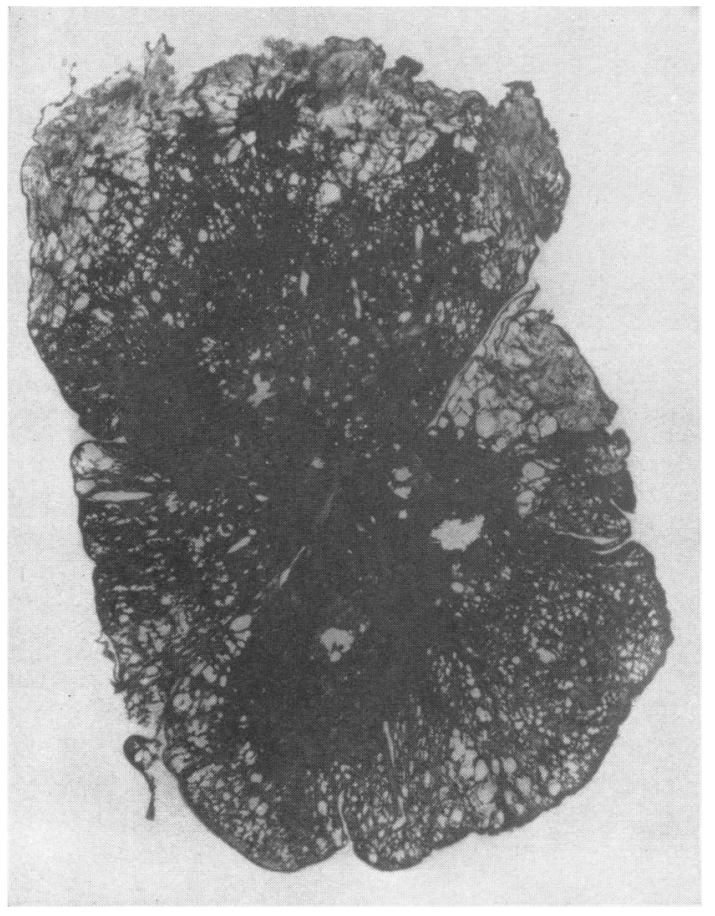

the type of disease produced is usually classical nodular silicosis; this series was analysed in order to provide a contrast to the pneumoconiosis of coal-miners.

The distribution of the new series in terms of working histories and degrees of pathology is shown in Table 1.

Coal varies in rank, and for South Wales' coals this variation can be adequately described by changes in the volatile matter content.

In conformity with the previous work we have ranked the coal as follows :-

\begin{tabular}{c|c|c}
\hline Symbol & \multicolumn{1}{|c|}{ Name } & Volatile Matter (\%) \\
\hline A & Anthracite & $4-10$ \\
S & Steam coal & $10-22$ \\
B & Bituminous coal & $22-36$ \\
\hline
\end{tabular}

Some workers had mixed histories in so far as they worked for several years in different types of coal.

Table 2 shows for the whole group from South Wales and for the more homogeneous group of colliers how many worked in coals of different rank.

It will be seen that most of the South Wales lungs came from persons who had worked in steam-coal 


\section{Methods of Analysis}

On removal from the body the whole right lung was cut into pieces and dried in an air oven at approximately $105^{\circ} \mathrm{C}$. in Professor Gough's laboratory at Cardiff. The dried lung was then sent to the Postgraduate Medical School of London. Here it was broken into small pieces, again dried at $105^{\circ} \mathrm{C}$., ground to a fine powder in a power-driven mortar, sieved through 60 B.S.S. screens, re-ground, and further dried to constant weight. It was then well mixed and sampled by "quartering", for determination of "coal " by the method of King and Gilchrist (1945) and ashed for chemical and $x$-ray analysis. For ashing, $50 \mathrm{~g}$. of the dried powder was placed in shallow "vitreosil" dishes (4 in. by 6 in.) and heated in a thermostat-controlled muffle furnace at $380^{\circ} \mathrm{C}$. until no black material remained. This required usually five to six days. The light fluffy ash which was free from carbon particles was now carefully brushed on to a $15 \mathrm{~cm}$. filter paper, Whatman No. 541 , by means of a camel hair brush. The filter paper was folded over the ash, placed inside a large Gooch filter and held in place by a wad of cotton wool. By means of a continuous liquid extractor the ash was washed twice with cold $\mathrm{N} \mathrm{HCl}$ for 24 hours and then washed with water. This was found to be adequate to remove completely all the $\mathrm{Fe}^{\prime \prime \prime}, \mathrm{PO}_{4}{ }^{\prime \prime \prime}$ and $\mathrm{CO}_{3}{ }^{\prime \prime}$ of the indigenous lung ash from the mineral dust and was not appreciably destructive of the siliceous minerals, as shown by the fact that only small amounts of silica were found in the $\mathrm{HCl}$ used for extraction (colorimetric method for silica, King, 1939).

The $\mathrm{HCl}$-insoluble residue of the ash consisted of the quartz and other siliceous minerals of the lung. The folded filter paper was removed from the Gooch filter, placed in a platinum crucible, dried, ignited at $380^{\circ} \mathrm{C}$. in the same muffle furnace as before, cooled, and weighed. This weight represented the "siliceous minerals" contained in the sample of dried lung. "Coal" plus "siliceous minerals" represents " total dust".

For silica analysis about $100 \mathrm{mg}$. of the siliceous minerals was fused with sodium carbonate, and the silica determined gravimetrically after double evaporation with hydrochloric acid by the classical method. The results were calculated to give the percentage $\mathrm{SiO}_{2}$ in the siliceous minerals and in the dried lung. Alumina was determined in the combined filtrate from the silica determination by the method of Gentry and Sherrington (1946).

The mineral content of the ashed acid-extracted lung residues was determined at the National Physical Laboratory and later also at the Safety in Mines Research Establishment by the $x$-ray diffraction technique using powder diagrams. In contrast to the previous results there was much less, and usually no kaolin in the residues. This was only discovered towards the end of the work. Check repeats with careful temperature control showed that several previously kaolin-free residues did contain kaolin when new samples were ashed at $380^{\circ} \mathrm{C}$., and it is likely that the bulk of the samples were ashed at too high a temperature. It was therefore not possible to make estimates of kaolin and mica separately but a joint " kaolin plus mica " estimate could be obtained in all cases. By visually estimating and comparing $x$-ray line intensities, the approximate relative proportions of quartz and kaolin plus mica were found and calculated back to percentages of dry lung on the basis of the total silica values.

\section{Results}

The results of the analyses are given in detail in Table 3. The lungs are grouped according to occupation and pathological grade as defined above, and, within each grade, according to increasing amounts of total dust as per cent. dry lung. Age, numbers of years worked in different occupations, and numbers of years elapsed between removal from dust and death are also given.

From the dry weights of the right lung given in the last column of Table 3 the actual amounts of dust in both lungs can be estimated by multiplying the percentages of the different constituents by twice the lung weights.

Effect of Occupation.-The composition of the dust varied with the type of occupation as shown in (A) Table 4 and Fig. 7 ; the occupations are arranged in increasing order of coal percentages of the lung dusts. The small groups of firemen, screen-workers, and non-miners are omitted from this tabulation, as there are only three lungs in each of the groups. "Coal " in tin-miners" and granite-workers" lungs is the ignitable portion of the residue prepared by alkali digestion. It is probably mainly soot from acetylene lamps, candles, and domestic fires, and
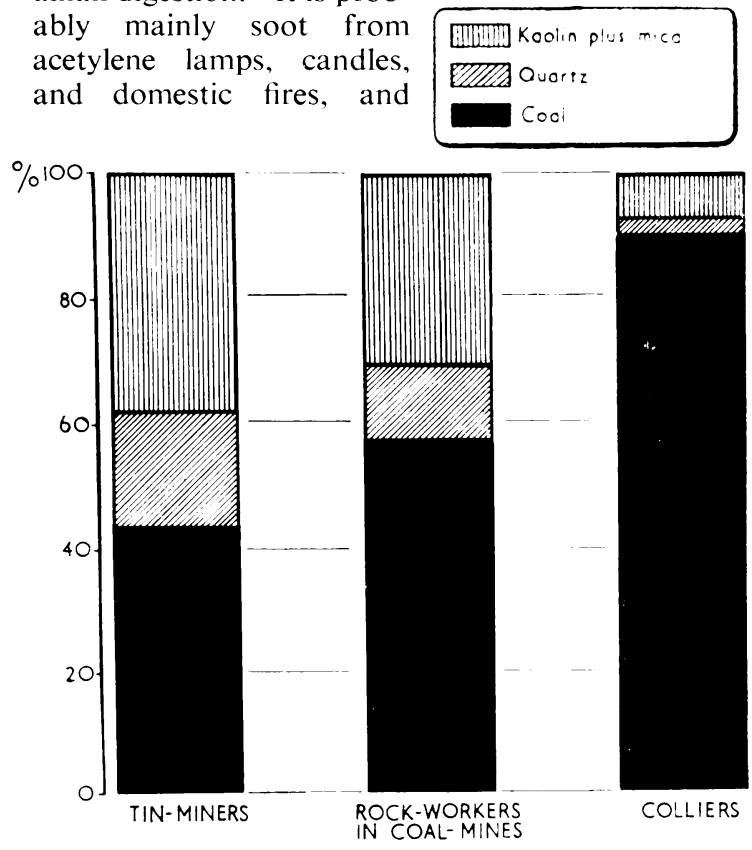

FIG. 7.- Relative proportions of coal, quartz, and kaolin plus mica in the average lung dust of different occupational groups. 
TABLE 3

MINERAL CONTENT OF LUNGS OF COALWORKERS AND TIN-MINERS

\begin{tabular}{|c|c|c|c|c|c|c|c|c|c|c|c|c|c|}
\hline \multirow[b]{2}{*}{ Chief Occupation } & \multirow[b]{2}{*}{$\begin{array}{l}\text { P.M. } \\
\text { No. }\end{array}$} & \multirow[b]{2}{*}{ Age } & \multicolumn{3}{|c|}{$\begin{array}{c}\text { Duration of } \\
\text { Exposure (years)* }\end{array}$} & \multirow{2}{*}{$\begin{array}{l}\text { Patho- } \\
\text { logical } \\
\text { Grade }\end{array}$} & \multicolumn{6}{|c|}{ Percentage Dry Lung } & \multirow{2}{*}{$\begin{array}{c}\text { Dry } \\
\text { Weight } \\
\text { of } \\
\text { Lung } \\
\text { (g.) }\end{array}$} \\
\hline & & & 1 & 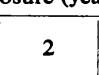 & 3 & & $\begin{array}{l}\text { Total } \\
\text { Dust }\end{array}$ & Coal & $\left|\begin{array}{c}\text { Sili- } \\
\text { ceous } \\
\text { Minerals }\end{array}\right|$ & Silica & Quartz & $\begin{array}{c}\text { Kaolin } \\
\text { plus } \\
\text { Mica }\end{array}$ & \\
\hline $\begin{array}{c}\text { I. Rock-workers } \\
\text { coal-mines }\end{array}$ in & $\begin{array}{l}51 \\
2 \\
35 \\
8 \\
75 \\
93 \\
94 \\
72 \\
77\end{array}$ & $\begin{array}{l}72 \\
58 \\
67 \\
67 \\
62 \\
66 \\
45 \\
62 \\
62\end{array}$ & $\begin{array}{l}20 \\
30 \\
50 \\
29 \\
26 \\
22 \\
18 \\
14 \\
35\end{array}$ & $\begin{array}{c}20 \text { (IV) } \\
\overline{ } \\
25 \overline{(I I I)} \\
15 \overline{\text { (II) }} \\
7 \text { (IV) } \\
16 \text { (III) } \\
3 \text { (IV) }\end{array}$ & $\begin{array}{l}2 \\
2 \\
2 \\
0 \\
? \\
1 \\
5 \\
5 \\
4\end{array}$ & $\begin{array}{l}1 \\
2 \\
3 \\
3 \\
3 \\
4 \\
4 \\
4 \\
4\end{array}$ & $\begin{array}{r}3.46 \\
5.40 \\
6 \cdot 21 \\
6 \cdot 36 \\
9 \cdot 27 \\
3 \cdot 18 \\
3.40 \\
8.34 \\
13.58\end{array}$ & $\begin{array}{l}2.20 \\
2.54 \\
3.20 \\
4.90 \\
5.90 \\
1.27 \\
1.75 \\
5.30 \\
6.50\end{array}$ & $\begin{array}{l}1.26 \\
2.86 \\
3.01 \\
1.46 \\
3.37 \\
1.91 \\
1.65 \\
3.04 \\
7.08\end{array}$ & $\begin{array}{l}0.84 \\
1.80 \\
2.03 \\
0.91 \\
2.15 \\
1.20 \\
0.99 \\
1.87 \\
4.40\end{array}$ & $\begin{array}{l}0.41 \\
0.92 \\
1.01 \\
0.42 \\
0.98 \\
0.48 \\
0.41 \\
0.75 \\
1.79\end{array}$ & $\begin{array}{l}0.86 \\
1.93 \\
2.01 \\
1.03 \\
2.38 \\
1.44 \\
1.22 \\
2.27 \\
5.28\end{array}$ & $\begin{array}{c}108 \\
133 \\
81 . \\
109 \\
138 \\
223 \\
245 \\
143 \\
205\end{array}$ \\
\hline II. Firemen & $\begin{array}{r}97 \\
9 \\
59\end{array}$ & $\begin{array}{l}71 \\
65 \\
54\end{array}$ & $\begin{array}{l}55+ \\
27 \\
18\end{array}$ & $\begin{array}{r}23 \overline{(I V)} \\
10(I V) \\
4 \text { (III) }\end{array}$ & $\begin{array}{l}0 \\
2 \\
5\end{array}$ & $\begin{array}{l}4 \\
4 \\
4\end{array}$ & $\begin{array}{r}5 \cdot 15 \\
14.12 \\
31 \cdot 41\end{array}$ & $\begin{array}{r}4 \cdot 10 \\
12 \cdot 2 \\
29 \cdot 50\end{array}$ & $\begin{array}{l}1.05 \\
1.92 \\
1.91\end{array}$ & $\begin{array}{l}0.70 \\
1.16 \\
1.22\end{array}$ & $\begin{array}{l}0.34 \\
0.45 \\
0.50\end{array}$ & $\begin{array}{l}0.72 \\
1.44 \\
1.43\end{array}$ & $\begin{array}{l}136 \\
189 \\
215\end{array}$ \\
\hline $\begin{array}{l}\text { III. Miscellaneous } \\
\text { underground } \\
\text { workers (hauliers, } \\
\text { repairers, road- } \\
\text { men) }\end{array}$ & $\begin{array}{l}34 \\
92 \\
89 \\
62 \\
63 \\
91 \\
27 \\
5 \\
44 \\
3\end{array}$ & $\begin{array}{l}51 \\
65 \\
69 \\
59 \\
60 \\
61 \\
65 \\
65 \\
72 \\
45\end{array}$ & $\begin{array}{l}30 \\
27 \\
16 \\
26 \\
34 \\
40 \\
44 \\
52 \\
42 \\
10\end{array}$ & $\begin{array}{c}5 \overline{(I V)} \\
6(\mathrm{I}) \\
6(\mathrm{IV}) \\
6(\mathrm{IV}) \\
6(\mathrm{IV}) \\
2(\mathrm{IV}) \\
16 \text { (IV) } \\
5 \text { (I) } \\
6 \text { (IV) }\end{array}$ & $\begin{array}{r}? \\
0 \\
10 \\
? \\
5 \\
2 \\
3 \\
0 \\
0 \\
0\end{array}$ & $\begin{array}{l}1 \\
1 \\
1 \\
1 \\
2 \\
2 \\
2 \\
3 \\
3 \\
5\end{array}$ & $\begin{array}{l}1.46 \\
1.79 \\
3.70 \\
3.72 \\
1 \cdot 10 \\
3.48 \\
4.81 \\
6.85 \\
8.62 \\
5.85\end{array}$ & $\begin{array}{l}1.17 \\
1.40 \\
3.50 \\
3.20 \\
0.90 \\
3.10 \\
3.20 \\
5.40 \\
5.90 \\
1.37\end{array}$ & $\begin{array}{l}0.29 \\
0.39 \\
0.20 \\
0.52 \\
0.20 \\
0.38 \\
1.61 \\
1.45 \\
2.72 \\
4.48\end{array}$ & $\begin{array}{l}0.18 \\
0.23 \\
0.13 \\
0.29 \\
0.13 \\
0.27 \\
1.04 \\
0.98 \\
1.75 \\
2.75\end{array}$ & $\begin{array}{l}0.10 \\
0.10 \\
0.06 \\
0.11 \\
0.06 \\
0.12 \\
0.41 \\
0.50 \\
0.83 \\
1.92\end{array}$ & $\begin{array}{l}0.17 \\
0.28 \\
0.14 \\
0.39 \\
0.14 \\
0.27 \\
1.21 \\
0.95 \\
1.89 \\
2.55\end{array}$ & $\begin{array}{r}128 \\
101 \\
130 \\
80 \\
63 \\
90 \\
114 \\
138 \\
137 \\
183 .\end{array}$ \\
\hline IV. Colliers & $\begin{array}{c}74 \\
82 \\
66 \\
17 \\
79 \\
25 / 26 \\
47 \\
53 \\
86 \\
7 \\
61 \\
57 \\
4 \\
6 \\
65 \\
108 \\
95 \\
45\end{array}$ & $\begin{array}{l}40 \\
49 \\
47 \\
42 \\
47 \\
60 \\
60 \\
50 \\
58 \\
53 \\
64 \\
45 \\
53 \\
55 \\
44 \\
56 \\
54 \\
53\end{array}$ & $\begin{array}{l}12 \\
12 \\
27 \\
14 \\
28 \\
30 \\
40 \\
36 \\
38 \\
31 \\
50 \\
23 \\
37 \\
25 \\
27 \\
27 \\
32 \\
24\end{array}$ & 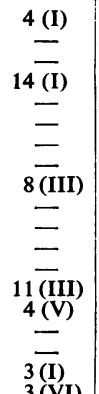 & $\begin{array}{r}4 \\
? \\
? \\
0 \\
7 \\
13 \\
2 \\
0 \\
2 \\
9 \\
9 \\
0 \\
5 \\
4 \\
7 \\
0 \\
16 \\
7 \\
10\end{array}$ & $\begin{array}{l}1 \\
2 \\
2 \\
2 \\
2 \\
2 \\
2 \\
2 \\
2 \\
3 \\
3 \\
3 \\
3 \\
3 \\
3 \\
3 \\
3 \\
3\end{array}$ & $\begin{array}{c}2.67 \\
2.33 \\
3.64 \\
4.13 \\
5.08 \\
5.22 \\
5.74 \\
6.57 \\
12.54 \\
7.19 \\
7.67 \\
8.23 \\
8.31 \\
11.47 \\
13.85 \\
16.0 \\
17.44 \\
21.07\end{array}$ & $\begin{array}{r}2.5 \\
2.0 \\
3.5 \\
3.5 \\
4.6 \\
4 \cdot 8 \\
4.4 \\
6 \cdot 0 \\
10 \cdot 2 \\
6.4 \\
5.5 \\
7 \cdot 3 \\
6 \cdot 4 \\
10.6 \\
12.5 \\
14.6 \\
16.1 \\
20.1\end{array}$ & $\begin{array}{l}0.17 \\
0.33 \\
0.14 \\
0.63 \\
0.48 \\
0.45 \\
1.34 \\
0.57 \\
2.34 \\
0.79 \\
2.17 \\
0.93 \\
1.87 \\
0.87 \\
1.35 \\
1.40 \\
1.34 \\
0.97\end{array}$ & $\begin{array}{l}0.11 \\
0.23 \\
0.09 \\
0.42 \\
0.31 \\
0.29 \\
0.81 \\
0.36 \\
1.42 \\
0.49 \\
1.32 \\
0.58 \\
1.08 \\
0.54 \\
0.89 \\
0.81 \\
0.78 \\
0.64\end{array}$ & $\begin{array}{l}0.04 \\
0.15 \\
0.04 \\
0.21 \\
0.13 \\
0.14 \\
0.33 \\
0.17 \\
0.58 \\
0.21 \\
0.52 \\
0.26 \\
0.41 \\
0.23 \\
0.40 \\
0.31 \\
0.29 \\
0.28\end{array}$ & $\begin{array}{l}0.13 \\
0.18 \\
0.11 \\
0.42 \\
0.34 \\
0.31 \\
1.01 \\
0.41 \\
1.75 \\
0.57 \\
1.65 \\
0.67 \\
1.45 \\
0.65 \\
0.96 \\
1.09 \\
1.04 \\
0.69\end{array}$ & $\begin{array}{r}98 \\
137 \\
136 \\
156 \\
189 \\
222 \\
130 \\
97 \\
98 \\
200 \\
131 \\
140 \\
155 \\
200 \\
158 \\
167 \\
187 \\
87\end{array}$ \\
\hline & $\begin{array}{r}107 \\
96 \\
99 \\
22 \\
1 \\
24 \\
10 \\
78 \\
52 \\
30\end{array}$ & $\begin{array}{l}54 \\
64 \\
63 \\
57 \\
64 \\
60 \\
59 \\
59 \\
59 \\
36\end{array}$ & $\begin{array}{l}24 \\
40 \\
42 \\
36 \\
19 \\
30 \\
29 \\
42 \\
43 \\
20\end{array}$ & $\begin{array}{c}7 \text { (VI) } \\
3 \text { (VI) } \\
\overline{\bar{O}} \\
9(\mathrm{VI}) \\
6(\mathrm{~V}) \\
11(\mathrm{II}) \\
\overline{\overline{(I I)}}\end{array}$ & $\begin{array}{r}10 \\
? \\
5 \\
7 \\
1 \\
1 \\
? \\
5 \\
6 \\
3 \\
2\end{array}$ & $\begin{array}{l}4 \\
4 \\
4 \\
4 \\
4 \\
4 \\
4 \\
4 \\
4 \\
4\end{array}$ & $\begin{array}{r}4 \cdot 36 \\
6 \cdot 46 \\
9 \cdot 15 \\
9 \cdot 33 \\
9.99 \\
19 \cdot 25 \\
19 \cdot 76 \\
21.84 \\
22.05 \\
25 \cdot 23\end{array}$ & $\begin{array}{l}3.5 \\
5.5 \\
8.9 \\
7.2 \\
6.9 \\
6.9 \\
18.4 \\
20.3 \\
20.1 \\
22.4 \\
23.4\end{array}$ & $\begin{array}{l}0.86 \\
0.96 \\
0.25 \\
2.13 \\
3.10 \\
0.85 \\
1.46 \\
1.74 \\
1.65 \\
1.83\end{array}$ & $\begin{array}{l}0.54 \\
0.59 \\
0.16 \\
1.26 \\
1.91 \\
0.53 \\
0.94 \\
1.07 \\
0.97 \\
1.13\end{array}$ & $\begin{array}{l}0.24 \\
0.26 \\
0.06 \\
0.47 \\
0.69 \\
0.25 \\
0.39 \\
0.41 \\
0.41 \\
0.43\end{array}$ & $\begin{array}{l}0.62 \\
0.70 \\
0.19 \\
1.65 \\
2.41 \\
0.59 \\
1.08 \\
1.33 \\
1.24 \\
1.41\end{array}$ & $\begin{array}{l}128 \\
151 \\
121 \\
140 \\
103 \\
106 \\
178 \\
200 \\
215 \\
296\end{array}$ \\
\hline V. Screen workers & $\begin{array}{r}123 \\
124 \\
69\end{array}$ & $\begin{array}{l}43 \\
55 \\
66\end{array}$ & $\begin{array}{l}21 \\
25 \\
-\end{array}$ & 二 & $\begin{array}{l}\frac{1}{7} \\
?\end{array}$ & $\begin{array}{l}1 \\
1 \\
2\end{array}$ & $\begin{array}{r}5.44 \\
10.87 \\
13.93\end{array}$ & $\begin{array}{r}4 \cdot 3 \\
10 \cdot 3 \\
12 \cdot 3\end{array}$ & $\begin{array}{l}1.14 \\
0.57 \\
1.68\end{array}$ & $\begin{array}{l}0.65 \\
0.34 \\
1.04\end{array}$ & $\begin{array}{l}0.31 \\
0.14 \\
0.43\end{array}$ & $\begin{array}{l}0.82 \\
0.43 \\
1.25\end{array}$ & $\begin{array}{l}116 \\
121 \\
109\end{array}$ \\
\hline $\begin{array}{l}\text { VI. Unclassified under- } \\
\text { ground }\end{array}$ & $\begin{array}{r}70 \\
15 \\
73 \\
20 \\
33 \\
21 \\
28 \\
12 \\
43 \\
48 \\
32 \\
90 \\
67 \\
58 \\
100 \\
42 \\
81 \\
76\end{array}$ & $\begin{array}{l}55 \\
59 \\
59 \\
44 \\
37 \\
77 \\
42 \\
53 \\
29 \\
65 \\
66 \\
59 \\
59 \\
25 \\
67 \\
73 \\
74 \\
54\end{array}$ & $\begin{array}{l}\frac{40}{30} \\
\frac{30}{17} \\
51 \\
35 \\
99 \\
39 \\
39 \\
46 \\
11 \\
= \\
40\end{array}$ & $\begin{array}{l}= \\
= \\
\bar{z} \\
5 \overline{(\mathrm{V})} \\
\bar{z} \\
\bar{z} \\
\bar{z} \\
= \\
=\end{array}$ & $\begin{array}{l}\frac{1}{5} \\
\frac{5}{5} \\
12 \\
0 \\
6 \\
51 \\
? 2 \\
2 \\
0 \\
0 \\
8 \\
9 \\
0\end{array}$ & $\begin{array}{l}1 \\
1 \\
1 \\
2 \\
2 \\
2 \\
2 \\
2 \\
2 \\
2 \\
3 \\
3 \\
3 \\
3 \\
3 \\
3 \\
4 \\
4\end{array}$ & $\begin{array}{r}1.26 \\
1.28 \\
9.16 \\
2.14 \\
3.25 \\
4.44 \\
4.85 \\
7.47 \\
9.20 \\
9.69 \\
2.52 \\
4.45 \\
4.50 \\
8.29 \\
12.22 \\
13.40 \\
9.88 \\
10.06\end{array}$ & $\begin{array}{c}1.00 \\
1.1 \\
7.9 \\
1.6 \\
2.2 \\
3.8 \\
3.7 \\
6.7 \\
7.7 \\
7.5 \\
2.0 \\
3.8 \\
2.8 \\
8.0 \\
9.2 \\
10.7 \\
5.4 \\
8.3\end{array}$ & $\begin{array}{l}0.26 \\
0.18 \\
1.26 \\
0.54 \\
1.02 \\
0.64 \\
1.19 \\
1.17 \\
1.50 \\
2.19 \\
0.52 \\
0.65 \\
1.70 \\
0.29 \\
3.02 \\
2.75 \\
4.48 \\
1.76\end{array}$ & $\begin{array}{l}0.16 \\
0.11 \\
0.80 \\
0.34 \\
0.58 \\
0.41 \\
0.78 \\
0.74 \\
0.96 \\
1.16 \\
0.29 \\
0.44 \\
1.10 \\
0.19 \\
1.80 \\
1.72 \\
2.82 \\
1.18\end{array}$ & $\begin{array}{l}0.07 \\
0.08 \\
0.36 \\
0.16 \\
0.24 \\
0.18 \\
0.38 \\
0.32 \\
0.45 \\
0.51 \\
0.13 \\
0.24 \\
0.50 \\
0.08 \\
0.75 \\
0.74 \\
1.25 \\
0.56\end{array}$ & $\begin{array}{l}0.19 \\
0.10 \\
0.90 \\
0.38 \\
0.78 \\
0.46 \\
0.81 \\
0.85 \\
1.04 \\
1.67 \\
0.39 \\
0.41 \\
1.20 \\
0.21 \\
2.28 \\
2.00 \\
3.22 \\
1.20\end{array}$ & $\begin{array}{l}67 \\
139 \\
140 \\
154 \\
159 \\
151 \\
228 \\
117 \\
77 \\
176 \\
169 \\
137 \\
104 \\
123 \\
128 \\
113 \\
166 \\
92\end{array}$ \\
\hline
\end{tabular}


Table 3 continued

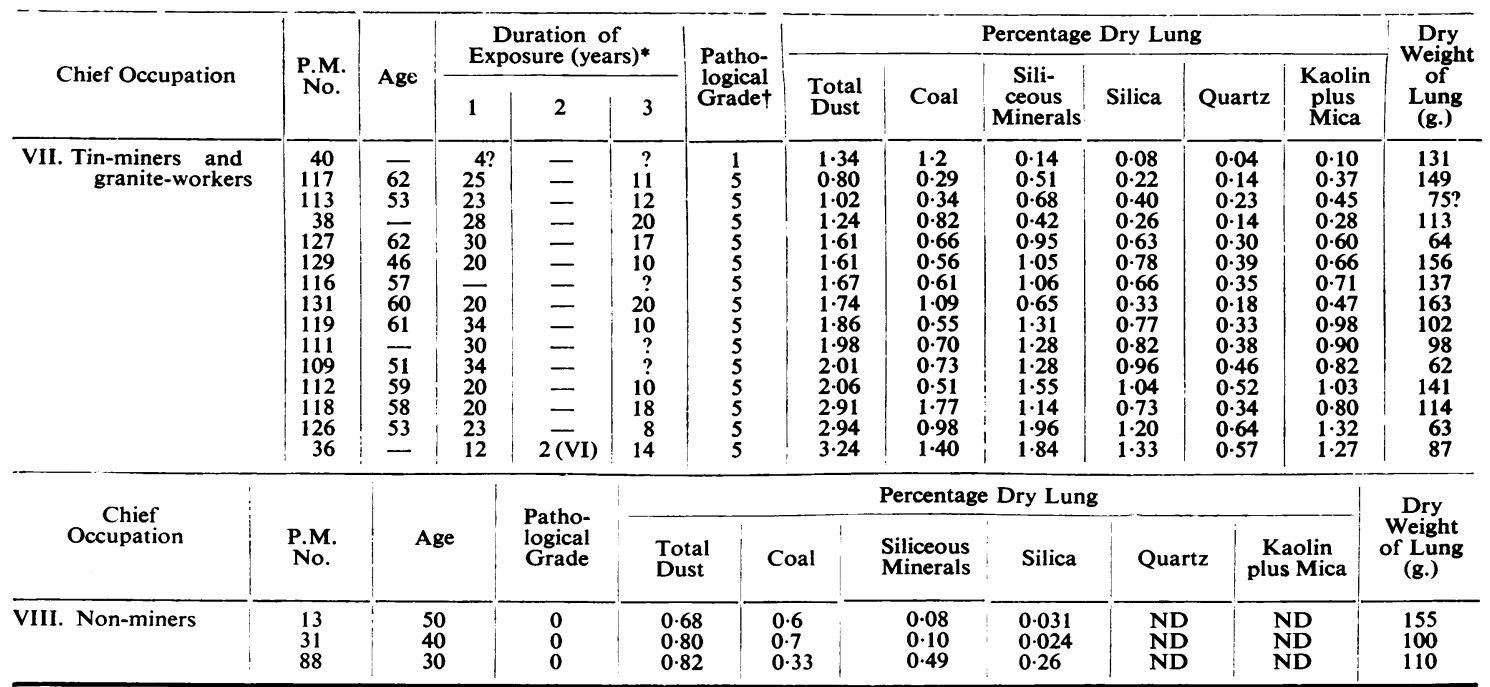

ND $=$ not determined.

* Duration of exposure : 1 =years spent in stated occupation ; $2=$ years spent in other underground occupation ; $3=$ years between removal from"dust and death.

† Pathological grade: $1=$ slight reticulation ; $2=$ reticulation ; $3=$ reticular and mixed nodulation ; $4=$ confluent fibrosis ; $5=$ silicotic nodulation.

TABLE 4

LUNG DUST COMPOSITION FOR DIFFERENT OCCUPATIONAL GROUPS FOR NEW AND OLD SERIES

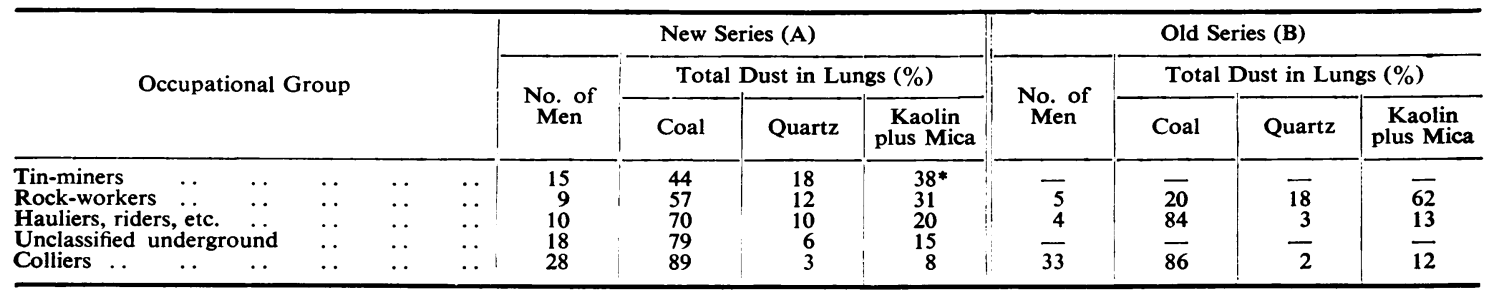

* Includes also felspar.

general atmospheric pollution. Likewise, the "coal" in the three normals probably represents atmospheric pollution. The average value of the three " normals", $1 \cdot 3 \mathrm{~g}$. of coal in both lungs, is slightly higher than the mean value for carbon in lungs of males recently given by Blacklock, Kennaway, Lewis, and Urquhart (1954) as $0.8 \mathrm{~g}$.

As one proceeds from tin-miners to rock-workers in coal-mines, hauliers, and finally colliers, the dust becomes richer in coal and decreases in siliceous minerals ; similar results obtained in the old series are shown in (B) Table 4 . In the new series the proportions of quartz to kaolin plus mica decrease slightly in the same order of occupations, but the differences are very small.

Thus the quartz percentages of the siliceous minerals in the lung residues are :
Tin-miners.. $\quad \ldots \quad \ldots \quad \ldots 32$

Rock-workers in coal-mines $\quad \ldots \quad 28$

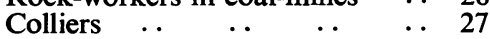

The dust as per cent. dry lung for different occupational groups is shown in Table 5 for total dust, coal, quartz, kaolin plus mica, and total silica. Here the differences between occupations are much more pronounced. Coalworkers' lungs as a class contain far more dust than tin-miners' or graniteworkers' lungs, but this difference is mainly due to coal itself. The proportions of quartz and silicates are about the same, except that the rock-workers in coal-mines have significantly more quartz and silicates than all other groups. It is worth while pointing out here that the quartz and silicate contents of the lungs of colliers are not significantly (at the $10 \%$ level) different from those of tin-miners. This will be discussed further. 
TABLE 5

LUNG DUST CONCENTRATION (\% DRY LUNG) FOR DIFFERENT OCCUPATIONAL GROUPS

\begin{tabular}{|c|c|c|c|c|c|c|c|c|c|}
\hline \multicolumn{4}{|c|}{ Occupation } & $\frac{\begin{array}{c}\text { No. of } \\
\text { Men }\end{array}}{15}$ & $\frac{\begin{array}{c}\text { Total Dust } \\
(\%)\end{array}}{1.87 \ldots 0.18^{*}}$ & $\frac{\begin{array}{c}\text { Coal } \\
\left(\begin{array}{c}0 \\
0\end{array}\right)\end{array}}{0.81 \quad 0.11}$ & $\underset{\left(0_{0}\right)}{\operatorname{Quartz}}$ & $\underset{\left(\begin{array}{c}0 \\
0\end{array}\right)}{\text { Kaolin plus Mica }}$ & $\frac{\begin{array}{c}\text { Total Silica } \\
(\%)\end{array}}{0.68: 0.09}$ \\
\hline $\begin{array}{l}\text { Tin-miners .. } \\
\text { Rock-workers } \\
\text { Hauliers, etc. } \\
\text { Unclassified .. } \\
\text { Colliers }\end{array}$ & $\begin{array}{l}\cdots \\
\cdots \\
\cdots \\
\cdots\end{array}$ & $\begin{array}{l}\ldots \\
\ldots \\
\cdots \\
\cdots\end{array}$ & $\begin{array}{l}\cdots \\
\cdots \\
\cdots \\
\cdots\end{array}$ & $\begin{array}{r}15 \\
9 \\
10 \\
18 \\
28\end{array}$ & $\begin{array}{r}1.87=0.18^{*} \\
6.58=1.13 \\
4.14=0.77 \\
6.56=0.90 \\
10.95=1.28\end{array}$ & 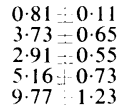 & $\begin{array}{ll}0.33 & 0.04 \\
0.80 & 0.15 \\
0.42 & 0.185 \\
0.39 & 0.07 \\
0.30 & 0.03\end{array}$ & $\begin{array}{lll}0.72 & 0.09 \dagger \\
2.05 & 0.44 \\
0.80 & 0.27 \\
1.00 & 0.20 \\
0.88 & 0.11\end{array}$ & $\begin{array}{lll}0.68 & 0.09 \\
1.80 & 0.37 \\
0.78 & 0.28 \\
0.87 & 0.16 \\
0.72 & 0.08\end{array}$ \\
\hline
\end{tabular}

* Standard error of mean. + Includes also felspar.

TABLE 6

ESTIMATED AMOUNTS OF TOTAL DUST AND VARIOUS CONSTITUENTS IN AVERAGE LUNGS OF DIFFERENT OCCUPATIONAL GROUPS

\begin{tabular}{|c|c|c|c|c|c|c|c|c|c|}
\hline \multicolumn{3}{|c|}{ Occupation } & $\begin{array}{l}\text { No. of } \\
\text { Men }\end{array}$ & $\begin{array}{c}\text { Mean Dust } \\
\text { Exposure } \\
\text { (years) }\end{array}$ & $\begin{array}{l}\text { Total } \\
\text { Dust } \\
\text { (g.) }\end{array}$ & $\begin{array}{c}\text { Coal } \\
\text { (g.) }\end{array}$ & $\begin{array}{l}\text { Quartz. } \\
\text { (g.) }\end{array}$ & $\begin{array}{c}\text { Kaolin plus } \\
\text { Mica } \\
\text { (g.) }\end{array}$ & $\begin{array}{l}\text { Total } \\
\text { Silica } \\
\text { (g.) }\end{array}$ \\
\hline $\begin{array}{l}\text { Tin-miners .. } \\
\text { Rock-workers } \\
\text { Hauliers, etc. .. } \\
\text { Unclassified } \ldots \\
\text { Colliers }\end{array}$ & $\begin{array}{l}\cdots \\
\cdots \\
\cdots \\
\cdots\end{array}$ & $\begin{array}{l}\cdots \\
\cdots \\
\cdots \\
\cdots\end{array}$ & $\begin{array}{r}15 \\
9 \\
10 \\
18 \\
28\end{array}$ & $\begin{array}{l}23 \\
37 \\
38 \\
34 \\
33\end{array}$ & $\begin{array}{c}4 \cdot 0=0 \cdot 4 \dagger \\
20 \cdot 2=4 \cdot 9 \\
10 \cdot 6-2 \cdot 5 \\
17 \cdot 4-2 \cdot 4 \\
34 \cdot 7 \quad 6 \cdot 2\end{array}$ & $\begin{array}{rr}1 \cdot 8 & 0 \cdot 3 \\
11 \cdot 1 & 2.4 \\
7.0 & 1.6 \\
13 \cdot 5 & 1.8 \\
31.1 & 5.8\end{array}$ & $\begin{array}{ll}0.7 & 0 \cdot 1 \\
2 \cdot 5 & 0.6 \\
1.3 & 0.7 \\
1 \cdot 1 & 0 \cdot 2 \\
0.9 & 0.1\end{array}$ & $\begin{array}{l:l}1 \cdot 5 & 0 \cdot 2+ \\
6 \cdot 6 & 2 \cdot 0 \\
2 \cdot 3 & 0 \cdot 9 \\
2 \cdot 8 & 0 \cdot 6 \\
2 \cdot 6 & 0.4\end{array}$ & $\begin{array}{l:l}1.4 & 0.2 \\
5.7 & 1.6 \\
2 \cdot 2 & 1.0 \\
2.4 & 0.5 \\
2.2 & 0.3\end{array}$ \\
\hline
\end{tabular}

* Sum of 1 and 2 in Table $3 .+$ Standard error of mean. \$ Contains also felspar.

Table 6 shows the estimated amount of total dust and its components present in both lungs. The figures and trends are similar to those in Table 5 but the spread of values is larger. Colliers' lungs contain on an average $35 \mathrm{~g}$. of dust, nearly nine times as much as is found in tin-miners' lungs. It will also be seen that colliers' lungs contain more quartz than the tin-miners' lungs (Fig. 8) although the difference is not statistically significant at the $10 \%$ level.

It should be noted that the lungs from Cornwall had been kept in formalin whereas those from South Wales were obtained fresh. It is possible that small amounts of dust may have been lost from the

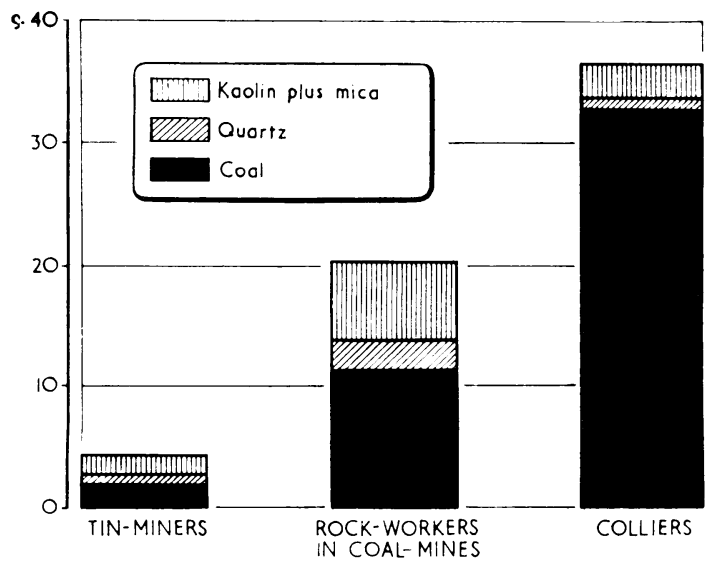

FIG. 8.-Amounts of dust constituents in average lungs of different occupational groups.
Cornish lungs, but such losses probably did not exceed $5 \%$ of the total dust.

Effect of Pathological Grade.-As mentioned in the introduction, the following pathological classification was used in conformity with the previous work.

\begin{tabular}{|c|c|c|}
\hline $\begin{array}{l}\text { Grade } \\
\text { No. }\end{array}$ & Description & $\begin{array}{c}\text { Approximate Radiographic } \\
\text { Equivalent }\end{array}$ \\
\hline $\begin{array}{l}1 \\
2 \\
3\end{array}$ & $\left.\begin{array}{l}\text { Slight reticulation } \\
\text { Reticulation } \\
\text { Mixed nodulation }\end{array}\right\}$ & Simple pneumoconiosis \\
\hline 4 & Confluent fibrosis & $\begin{array}{l}\text { Progressive massive } \\
\text { fibrosis }\end{array}$ \\
\hline 5 & Silicotic nodulation & \\
\hline
\end{tabular}

Grade 3 corresponds mainly to simple pneumoconiosis, but may also contain cases with " infective" nodules.

This classification is mainly based on the type of lesion revealed by study of macro- and microsections and not necessarily on the amount of fibrotic tissue. The radiographic equivalents are based on the data given in Table 12.

Taking all cases together, Table 7 shows the mean values for coal, quartz, silicates, and also total silica as percentages of dry lung. There is a regular increase from grade 1 to 4 although the differences between grade 3 and 4 and also the increase in coal content from grade 1 to 2 are not statistically significant at the $10 \%$ level. The actual significance levels of the differences are listed in Table 7a. If a difference is statistically significant at, say, the $1 \%$ level, it means that there is less than one chance in a hundred that such a difference could have arisen due to chance alone. 
TABLE 7

DUST CONCENTRATION IN RELATION TO PATHOLOGICAL GRADE FOR ALL CASES

\begin{tabular}{|c|c|c|c|c|c|c|c|}
\hline \multirow[b]{2}{*}{$\begin{array}{l}\text { Patho- } \\
\text { logical } \\
\text { Grade }\end{array}$} & \multirow[b]{2}{*}{$\begin{array}{c}\text { No. of } \\
\text { Men }\end{array}$} & \multirow{2}{*}{$\begin{array}{c}\text { Mean } \\
\text { Dust } \\
\text { Expo- } \\
\text { sure } \\
\text { (years)* }\end{array}$} & \multicolumn{5}{|c|}{ Percentage Dry Lung } \\
\hline & & & $\begin{array}{l}\text { Total } \\
\text { Dust } \\
(\%)\end{array}$ & $\begin{array}{l}\text { Coal } \\
(\%)\end{array}$ & $\underset{(\%)}{\text { Quartz }}$ & $\begin{array}{c}\text { Kaolin } \\
\text { plus } \\
\text { Mica } \\
(\%)\end{array}$ & $\begin{array}{c}\text { Total } \\
\text { Silica } \\
(\%)\end{array}$ \\
\hline $\begin{array}{l}1 \\
2 \\
3 \\
4 \\
5\end{array}$ & $\begin{array}{l}12 \\
20 \\
20 \\
19 \\
15\end{array}$ & $\begin{array}{l}26 \\
33 \\
38 \\
37 \\
24\end{array}$ & $\begin{array}{r}3.85 \\
5.75 \\
9.70 \\
12.98 \\
2.17\end{array}$ & $\begin{array}{r}3 \cdot 31 \\
4.69 \\
8 \cdot 06 \\
10 \cdot 89 \\
0.83\end{array}$ & $\begin{array}{l}0.15 \\
0.30 \\
0.45 \\
0.53 \\
0.46\end{array}$ & $\begin{array}{l}0.38 \\
0.77 \\
1.18 \\
1.55 \\
0.88\end{array}$ & $\begin{array}{l}0.33 \\
0.66 \\
1.02 \\
1.30 \\
0.86\end{array}$ \\
\hline
\end{tabular}

* Sum of 1 and 2 in Table 3.

TABLE 7a

SIGNIFICANCE LEVELS OF DIFFERENCES OF MEAN VALUES GIVEN IN TABLE 7

\begin{tabular}{|c|c|c|c|c|c|}
\hline \multirow{2}{*}{$\begin{array}{l}\text { Difference of } \\
\text { Pathological } \\
\text { Grades }\end{array}$} & \multicolumn{5}{|c|}{ Significance Level (\%) for } \\
\hline & $\begin{array}{l}\text { Total } \\
\text { Dust }\end{array}$ & Coal & Quartz & $\begin{array}{c}\text { Kaolin } \\
\text { plus } \\
\text { Mica }\end{array}$ & Silica \\
\hline $\begin{array}{l}2-1 \\
3-2 \\
4-3 \\
4-2\end{array}$ & $\begin{array}{l}\text { NS } \\
1 \\
\text { NS } \\
0 \cdot 1\end{array}$ & $\begin{array}{l}\text { NS } \\
2 \\
\text { NS } \\
1\end{array}$ & $\begin{array}{c}5 \\
5 \\
\text { NS } \\
5\end{array}$ & $\begin{array}{c}5 \\
5 \\
\text { NS } \\
1\end{array}$ & $\begin{array}{c}5 \\
5 \\
\text { NS } \\
2\end{array}$ \\
\hline
\end{tabular}

$\mathrm{NS}=$ not significant at the $10 \%$ level.

A co-variance analysis was carried out to investigate the effect of exposure time on the quartz and coal dust content of the lungs. As the exposure times did not have a sufficiently large range, the exposure effect was not significant at the $10 \%$ level. The analysis did, however, confirm that in general there are significant differences between grades. It is quite obvious that grade 5 , the silicotic nodulation, does not fit into the picture of regular increase of dust concentration with increasing grade of pathology.

The work described in this report had been undertaken in continuation of the previous analyses in the hope that the data could be pooled and thus more significant results be obtained. On comparing the average results (percentages of dry lung) for the different pathological groups it was found, however, that the new results were in general considerably lower than the old ones. The data for all cases, namely 54 lungs in the old and 87 in the new series, are assembled in Table 8 and for the more homogeneous groups of colliers (27 in each series) in Table 9.

The differences between the two series are probably due to selection of lung material. For the old series representative fibrotic areas of the lungs had been chosen and it appears very likely that lower dust percentages would have been found had a whole lung been analysed as was done in the new series. There is, however, another difference between the two series. The lungs in the old series came chiefly from anthracite-workers, whereas in the new series they came chiefly from steam-coal workers. It appears to us very unlikely that this should account for lower dust proportions at a given pathological stage, as it would make a unit of steam-coal dust more pathogenic than a unit of anthracite dust and no trace of such an effect had been found in the old series; nevertheless it points to a gap in our knowledge.

It is intended to remedy this deficiency by analysing, in the future, more lungs from anthracite and bituminous areas of South Wales as well as from other English and Scottish coalfields. For the present it was felt better, however, to restrict a detailed evaluation to the results of the new series.

Ignoring for the moment the differences in level of dust concentrations between the old and new series, it will be seen from Tables 8 and 9 that the trends for increasing proportions of dust with increasing grade of pathology are similar in both series for the grades 1 to 4 . Grade 5 is not comparable at all. In the old series three rock-workers in coal-mines with very high silica content of the lungs were classed as grade 5, whereas in the new series there is only one repairer accompanied by 15 tin-miners and granite-workers in this grade (classical silicosis).

TABLE 8

DUST CONCENTRATION IN RELATION TO PATHOLOGICAL GRADE FOR ALL CASES AND MEAN VALUES OF DUST FOR THE OLD AND NEW SERIES

\begin{tabular}{|c|c|c|c|c|c|c|c|c|c|c|c|c|c|c|}
\hline \multirow{3}{*}{$\begin{array}{l}\text { Pathological } \\
\text { Grade }\end{array}$} & \multirow{2}{*}{\multicolumn{2}{|c|}{$\begin{array}{l}\text { No. of } \\
\text { Lungs }\end{array}$}} & \multirow{2}{*}{\multicolumn{2}{|c|}{$\begin{array}{c}\text { Exposure } \\
\text { (years) }\end{array}$}} & \multicolumn{10}{|c|}{ Percentage Dry Lung } \\
\hline & & & & & \multicolumn{2}{|c|}{ Total Dust } & \multicolumn{2}{|c|}{ Coal } & \multicolumn{2}{|c|}{ Quartz } & \multicolumn{2}{|c|}{ Kaolin plus Mica } & \multicolumn{2}{|c|}{ Silica } \\
\hline & Old & New & Old & New & Old & New & Old & New & Old & New & Old & New & Old & New \\
\hline $\begin{array}{l}1 \\
2 \\
3 \\
4 \\
5\end{array}$ & $\begin{array}{r}\overline{14} \\
13 \\
24 \\
3\end{array}$ & $\begin{array}{l}12 \\
20 \\
20 \\
19 \\
15\end{array}$ & $\begin{array}{l}-\overline{38} \\
38 \\
31 \\
18\end{array}$ & $\begin{array}{l}26 \\
33 \\
38 \\
37 \\
24\end{array}$ & $\begin{array}{r}-\overline{12 \cdot 47} \\
13 \cdot 79 \\
16 \cdot 52 \\
4 \cdot 76\end{array}$ & $\begin{array}{r}3.85 \\
5.75 \\
9 \cdot 70 \\
12.98 \\
2.17\end{array}$ & $\begin{array}{r}10 \overline{71} \\
11.42 \\
14.25 \\
0.73\end{array}$ & $\begin{array}{r}3.31 \\
4.69 \\
8.06 \\
10.89 \\
0.83\end{array}$ & $\begin{array}{l}\overline{0.28} \\
0 \cdot 34 \\
0.37 \\
0.96\end{array}$ & $\begin{array}{l}0 \cdot 15 \\
0.30 \\
0.45 \\
0 \cdot 53 \\
0.46\end{array}$ & $\begin{array}{l}1.48 \\
2.03 \\
1.90 \\
3.07\end{array}$ & $\begin{array}{l}0 \cdot 38 \\
0.77 \\
1 \cdot 18 \\
1.55 \\
0.88\end{array}$ & $\begin{array}{l}\overrightarrow{0.94} \\
1.25 \\
1.23 \\
2.34\end{array}$ & $\begin{array}{l}0.33 \\
0.66 \\
1.02 \\
1.30 \\
0.86\end{array}$ \\
\hline
\end{tabular}

Italicized values are different at $10 \%$ significance level comparing old and new series. Bold type values are different at $1 \%$ significance level comparing old and new series. 
TABLE 9

DUST CONCENTRATION IN RELATION TO PATHOLOGICAL GRADE FOR COLLIERS ONLY AND MEAN VALUES OF DUST FOR THE OLD AND NEW SERIES

\begin{tabular}{|c|c|c|c|c|c|c|c|c|c|c|c|c|c|c|}
\hline \multirow{3}{*}{$\begin{array}{l}\text { Pathological } \\
\text { Grade }\end{array}$} & \multirow{2}{*}{\multicolumn{2}{|c|}{$\begin{array}{l}\text { No. of } \\
\text { Lungs }\end{array}$}} & \multirow{2}{*}{\multicolumn{2}{|c|}{$\begin{array}{c}\text { Exposure } \\
\text { (years) }\end{array}$}} & \multicolumn{10}{|c|}{ Percentage Dry Lung } \\
\hline & & & & & \multicolumn{2}{|c|}{ Total Dust } & \multicolumn{2}{|c|}{ Coal } & \multicolumn{2}{|c|}{ Quartz } & \multicolumn{2}{|c|}{ Kaolin plus Mica } & \multicolumn{2}{|c|}{ Silica } \\
\hline & Old & New & Old & New & Old & New & Old & New & Old & New & Old & New & Old & New \\
\hline $\begin{array}{l}2 \\
3 \\
4\end{array}$ & $\begin{array}{r}8 \\
7 \\
12\end{array}$ & $\begin{array}{r}8 \\
9 \\
10\end{array}$ & $\begin{array}{l}41 \\
36 \\
35\end{array}$ & $\begin{array}{l}30 \cdot 9 \\
33 \cdot 0 \\
36 \cdot 2\end{array}$ & $\begin{array}{l}14.59 \\
14.65 \\
18.12\end{array}$ & $\begin{array}{r}5 \cdot 66 \\
12 \cdot 36 \\
14 \cdot 74\end{array}$ & $\begin{array}{l}12 \cdot 78 \\
12 \cdot 30 \\
16.06\end{array}$ & $\begin{array}{r}4.87 \\
11.06 \\
13.26\end{array}$ & $\begin{array}{l}0 \cdot 25 \\
0 \cdot 32 \\
0 \cdot 31\end{array}$ & $\begin{array}{l}0.22 \\
0.32 \\
0.36\end{array}$ & $\begin{array}{l}1.57 \\
2.03 \\
1.74\end{array}$ & $\begin{array}{l}0.57 \\
0.97 \\
1.1: 2\end{array}$ & $\begin{array}{l}0.95 \\
1.23 \\
1.10\end{array}$ & $\begin{array}{l}0.4 ! \\
0.79 \\
0.91\end{array}$ \\
\hline
\end{tabular}

It alicized values are different at $10 \%$ at least significance level comparing old and new series. Bold type values are different at $1 \%$ at least significance level comparing old and new series.

TABLE 10

ESTIMATED AVERAGE AMOUNT OF TOTAL DUST AND VARIOUS CONSTITUENTS IN BOTH LUNGS OF MEN FROM DIFFERENT PATHOLOGICAL GRADES FOR ALL CASES

\begin{tabular}{|c|c|c|c|c|c|c|c|}
\hline $\begin{array}{l}\text { Patho- } \\
\text { logical } \\
\text { Grade }\end{array}$ & $\begin{array}{c}\text { No. of } \\
\text { Men }\end{array}$ & $\begin{array}{l}\text { Mean } \\
\text { Dust } \\
\text { Expo- } \\
\text { sure } \\
\text { (years)* }\end{array}$ & $\begin{array}{c}\text { Total } \\
\text { Dust } \\
\text { (g.) }\end{array}$ & $\begin{array}{c}\text { Coal } \\
\text { (g.) }\end{array}$ & $\underset{\text { (g.) }}{\text { Quartz }}$ & $\begin{array}{c}\text { Kaolin } \\
\text { plus } \\
\text { Mica } \\
\text { (g.) }\end{array}$ & $\begin{array}{c}\text { Total } \\
\text { Silica } \\
\text { (g.) }\end{array}$ \\
\hline $\begin{array}{l}1 \\
2 \\
3 \\
4 \\
5\end{array}$ & $\begin{array}{l}12 \\
20 \\
20 \\
19 \\
15\end{array}$ & $\begin{array}{l}26 \\
33 \\
38 \\
37 \\
24\end{array}$ & $\begin{array}{r}9 \cdot 1 \\
15 \cdot 3 \\
27 \cdot 3 \\
47 \cdot 7 \\
5 \cdot 2\end{array}$ & $\begin{array}{r}7.9 \\
12.4 \\
23.0 \\
40 \cdot 3 \\
1.9\end{array}$ & $\begin{array}{l}0.35 \\
0.80 \\
1.19 \\
1.88 \\
1.15\end{array}$ & $\begin{array}{l}0.86 \\
2.06 \\
3 \cdot 15 \\
5.53 \\
2.09\end{array}$ & $\begin{array}{l}0.76 \\
1.77 \\
2.71 \\
4.60 \\
2.05\end{array}$ \\
\hline
\end{tabular}

* Sum of 1 and 2 in Table 3.

TABLE 10a

SIGNIFICANCE LEVELS OF DIFFERENCES OF MEAN VALUES GIVEN IN TABLE 10

\begin{tabular}{|c|c|c|c|c|c|}
\hline \multirow{2}{*}{$\begin{array}{l}\text { Difference of } \\
\text { Pathological } \\
\text { Grades }\end{array}$} & \multicolumn{5}{|c|}{ Significance Level $\left(\sigma_{0}\right)$ for } \\
\hline & $\begin{array}{l}\text { Total } \\
\text { Dust }\end{array}$ & Coal & Quartz & $\begin{array}{c}\text { Kaolin } \\
\text { plus } \\
\text { Mica }\end{array}$ & Silica \\
\hline $2-1$ & 10 & 10 & 5 & 5 & 2 \\
\hline $3-2$ & 1 & 1 & 5 & 5 & 5 \\
\hline $4-3$ & 5 & 10 & 10 & 5 & 5 \\
\hline $4-2$ & 0.1 & 1 & I & $i$ & 1 \\
\hline
\end{tabular}

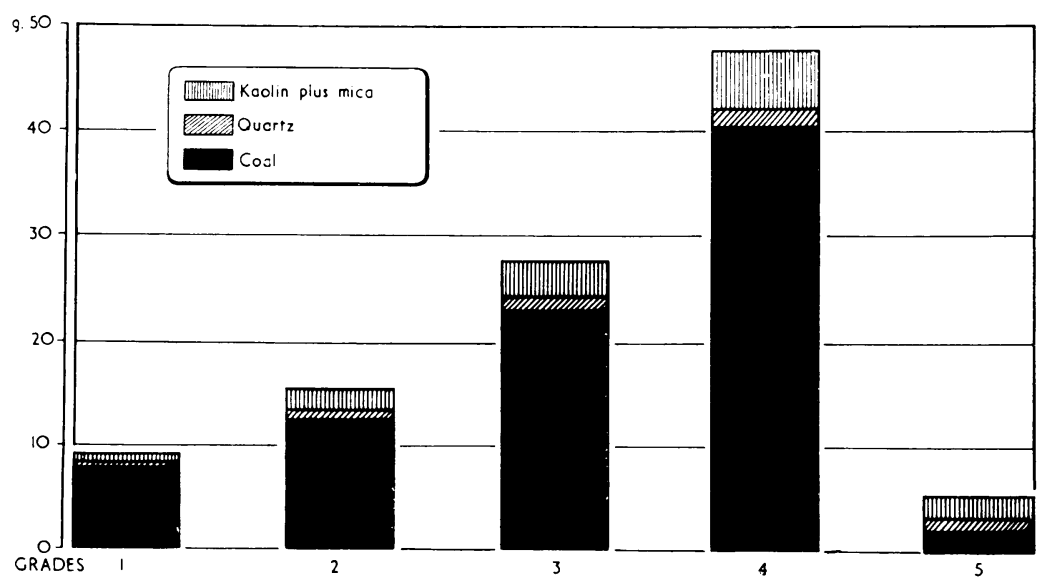

FIG. 9.-Total dust in lungs for different pathological grades (all cases).
Mean values of total dust in lungs for the different pathological groups are shown in Table 10 and Fig. 9. The Table shows the same trends as Table 7, but in a more pronounced form, because the lungs tend to become heavier with increasing pathological grade. Nevertheless, even on the basis of total dust, quartz, or silica the increase from grade 3 to grade 4 is not statistically significant; this is due to the large variations found within each pathological group and especially in grade 4. Table 11 and Fig. 10 show similar data for the more homogeneous group of colliers.

The increase in lung weight with increasing pathological grading is significant (at the $1 \%$ level) and is not entirely accounted for by the dust itself, as may be seen from the following figures :-

Grade 4 lungs are on an average $20 \mathrm{~g}$. heavier than those of grades 2 and 3 . This increase in weight is probably mainly due to fibrotic tissue and the main constituent of such tissue is collagen. A technique of collagen determination, recently developed for use in animal experiments (Stacy and King, 1954) was used on average samples of dried lung from the different pathological grades. The following results were obtained :-

The difference in collagen between grade 4 and the average of grades 1 , 2 , and 3 is of the order of $6 \mathrm{~g}$.

Dense fibrotic lung tissue of rats produced in experimental silicosis was found to contain $40 \%$ of collagen. Using this value, $6 \mathrm{~g}$. of collagen correspond to $15 \mathrm{~g}$. of fibrotic tissue, in reasonable agreement with the $20 \mathrm{~g}$. increase in weight of dust-free lungs of grade 4 over those of grades 1, 2, and 3 . 
TABLE 11

ESTIMATED AVERAGE AMOUNT OF TOTAL DUST AND VARIOUS CONSTITUENTS IN BOTH LUNGS OF MEN FROM DIFFERENT PATHOLOGICAL GRADES FOR COLLIERS ONLY

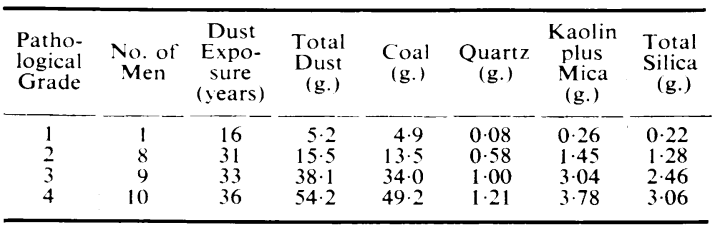

TABLE 1la

SIGNIFICANCE LEVELS OF DIFFERENCES OF MEAN VALUES GIVEN IN TABLE 11

\begin{tabular}{|c|c|c|c|c|c|}
\hline \multirow{2}{*}{$\begin{array}{c}\text { Difference of } \\
\text { Pathological } \\
\text { Grades }\end{array}$} & \multicolumn{4}{|c|}{ Significance Level $\left({ }^{\circ}{ }_{0}\right)$ for } & \multirow[b]{2}{*}{ Silica } \\
\hline & $\begin{array}{l}\text { Total } \\
\text { Dust }\end{array}$ & Coal & Quart $z$ & $\begin{array}{c}\text { Kaolin } \\
\text { plus } \\
\text { Mica }\end{array}$ & \\
\hline $3-2$ & 1 & 1 & 2 & 1 & 1 \\
\hline $4-3$ & NS & NS & NS & NS & NS \\
\hline 42 & 5 & 5 & 5 & 5 & 5 \\
\hline
\end{tabular}

NS not significant at the $10^{\circ}{ }^{\circ}$ le:el.

Radiography.-A comparison of Table 3 with the similar data given for the old series (Table 1 in King and Nagelschmidt, 1945) will show that $x$-ray data have been omitted, although $x$-ray assessments were available for some but not all the men in the new series. These radiographs had been taken between 1944 and 1947 under conditions which would not now be regarded as satisfactory. An attempt has been made to read these old radiographs by experienced radiologists of the Pneumoconiosis Research Unit, Cardiff, and the results are given in Table 14. They show a general parallelism between amount of dust, pathological grading, and radiological reading, and they also show that, with one exception, the pathological grades 1 to 3 used in this paper correspond to radiological diagnosis of simple pneumoconiosis and grade 4 to progressive massive fibrosis as might be expected. But the information is too scanty to be analysed in detail. It is hoped in future work to make more detailed comparison between satisfactory radiographs, a new pathological classification, and detailed lung dust analyses.

Size of Lung Dust.-Some preliminary work on measurement of the particle size distribution of the lung residues has been done (Cartwright and Nagelschmidt, 1951). The techniques developed, using light microscopy only, were not entirely satisfactory and further study by light and electron microscopy is required. Trypsin digestion was found to be the least destructive method of isolating the lung dust, and size distributions of the mean of
TABLE 12

WEIGHTS OF DUST AND LUNGS FOR DIFFERENT PATHOLOGICAL GRADES

\begin{tabular}{cccc}
\hline $\begin{array}{c}\text { Pathological } \\
\text { Grade }\end{array}$ & $\begin{array}{c}\text { Mean Dry } \\
\text { Weight of } \\
\text { Right Lung } \\
\text { (g.) }\end{array}$ & $\begin{array}{c}\text { Mean Total } \\
\text { Dust in } \\
\text { Right Lung } \\
\text { (g.) }\end{array}$ & $\begin{array}{c}\text { Mean Weight } \\
\text { of Right Lung } \\
\text { Minus Dust } \\
\text { (g.) }\end{array}$ \\
\hline 0 & 111 & 1.1 & 110 \\
1 & 113.3 & 4.6 & $108 \cdot 7$ \\
2 & 136.8 & 7.6 & 129.2 \\
3 & 140.1 & 13.7 & 126.4 \\
4 & 171.3 & 24.0 & 147.3 \\
5 & 113.1 & 2.2 & 110.9 \\
\hline
\end{tabular}

TABLE 13

COLLAGEN IN LUNGS OF DIFFERENT PATHOLOGICAL GRADES

\begin{tabular}{ccc}
\hline $\begin{array}{c}\text { Pathological } \\
\text { Grade }\end{array}$ & $\begin{array}{c}\text { Collagen in Dried } \\
\text { Lung }\left(\begin{array}{c}0 \\
0\end{array}\right)\end{array}$ & $\begin{array}{c}\text { Collagen in Dust-free } \\
\text { Right Lung (g.) }\end{array}$ \\
\hline 0 & $5 \cdot 0$ & $5 \cdot 5$ \\
1 & $5 \cdot 6$ & $6 \cdot 1$ \\
2 & $4 \cdot 5$ & $5 \cdot 8$ \\
3 & $5 \cdot 2$ & $6 \cdot 6$ \\
4 & $8 \cdot 3$ & $12 \cdot 2$ \\
5 & $7 \cdot 5$ & $8 \cdot 3$ \\
\hline
\end{tabular}

three lung dusts are shown in Fig. 6. The size of the coal is generally larger than the size of the non-coal. This has recently been confirmed by electron microscope observations (Policard and Collet, 1953).

\section{Discussion}

In the preceding sections the analytical data for the dust found in the lungs of 71 pneumoconiotic workers from South Wales and of 15 silicotic tinminers and granite-workers from Cornwall have been presented. It remains to be seen what information can be derived from the results which might throw

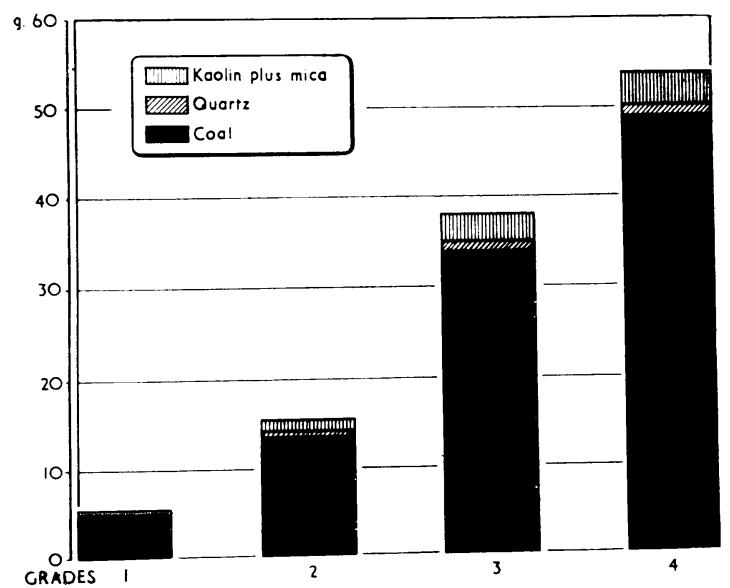

Fig. 10.-Total dust in lungs for different pathological grades (colliers only). 
TABLE 14

RADIOLOGICAL ASSESSMENT OF SELECTED LUNGS IN RELATION TO PATHOLOGICAL GRADE AND TOTAL DUST

\begin{tabular}{|c|c|c|c|}
\hline $\begin{array}{l}\text { Necropsy } \\
\text { No. }\end{array}$ & $\begin{array}{c}\text { Pathological } \\
\text { Grade }\end{array}$ & $\begin{array}{c}X \text {-ray Reading } \\
\text { (P.R.U. } \\
\text { Classification) }\end{array}$ & $\begin{array}{c}\text { Total Dust } \\
\text { (\% Dry Lung) }\end{array}$ \\
\hline $\begin{array}{r}70 \\
92 \\
62 \\
63 \\
20 \\
91 \\
17 \\
27 \\
2 \\
47 \\
53 \\
12 \\
48 \\
86 \\
90 \\
7 \\
57 \\
4 \\
44 \\
95 \\
93 \\
107 \\
96 \\
72 \\
77 \\
9 \\
10 \\
78 \\
52 \\
59\end{array}$ & $\begin{array}{l}1 \\
1 \\
1 \\
2 \\
2 \\
2 \\
2 \\
2 \\
2 \\
2 \\
2 \\
2 \\
2 \\
2 \\
3 \\
3 \\
3 \\
3 \\
3 \\
3 \\
4 \\
4 \\
4 \\
4 \\
4 \\
4 \\
4 \\
4 \\
4 \\
4\end{array}$ & 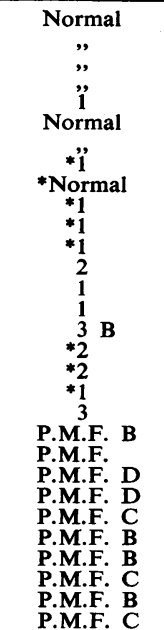 & \begin{tabular}{|r|}
$1 \cdot 2$ \\
$1 \cdot 8$ \\
$3 \cdot 7$ \\
$1 \cdot 1$ \\
$2 \cdot 1$ \\
$3 \cdot 5$ \\
$4 \cdot 1$ \\
$4 \cdot 8$ \\
$5 \cdot 4$ \\
$5 \cdot 7$ \\
$6 \cdot 6$ \\
$7 \cdot 5$ \\
$9 \cdot 7$ \\
$12 \cdot 5$ \\
$4 \cdot 4$ \\
$7 \cdot 2$ \\
$8 \cdot 2$ \\
$8 \cdot 3$ \\
$4 \cdot 8$ \\
$17 \cdot 4$ \\
$3 \cdot 2$ \\
$4 \cdot 4$ \\
$6 \cdot 5$ \\
$8 \cdot 3$ \\
$13 \cdot 6$ \\
$14 \cdot 0$ \\
$19 \cdot 8$ \\
$21 \cdot 8$ \\
$22 \cdot 0$ \\
31.4
\end{tabular} \\
\hline
\end{tabular}

*Assessment doubtful in view of poor quality of film.

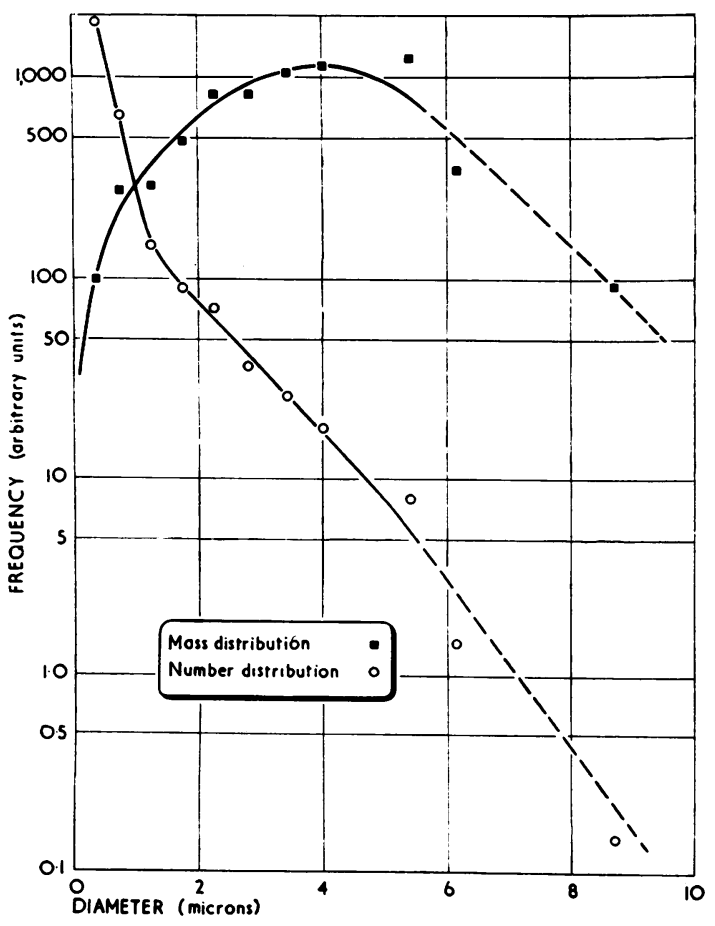

Fig. 6. - Number and mass distribution of trypsin digested residues. light on the aetiology and method of progression of the disease. It should be borne in mind that the sample of lungs was obtained from post-mortem material from March, 1946, to March, 1947. The lungs were collected until equal numbers of different pathological grades were obtained, but the cases used were not necessarily consecutive ones in any one grade, as collection had to be adjusted to the available facilities for drying the lungs, and in general only those cases were used where senior staff had carried out the necropsy. Of all cases occurring in the period of one year, roughly half were used for grade 1, half for grade 2, about two-thirds for grade 3, and a little more than one-fifth for grade 4.

The assessment of the pathological grade is considered to be entirely reliable for grade 4 and to have a very small observer error for the other grades (Gough, private communication). .

Statistically the source of data is not completely satisfactory. It would have been better if the cases could have been selected at random out of those available in the period of a year. However, this was not possible for the reasons stated. Nevertheless, it was assumed in analysing the data that the cases used were a random sample from the population of post-mortem cases.

A great difficulty in interpreting the results is the large variability of the data even within given occupational and pathological groups. For instance, colliers with fibrosis classed as grade 4 ranged in age from 36 to 64 years, and in working time from 21 to 43 years' dust exposure. One man collected $150 \mathrm{~g}$. of dust in 21 years of dust exposure, another $20 \mathrm{~g}$. in over 40 years, while a third collected $70 \mathrm{~g}$. of dust in 40 years. Similar examples can be given for grade 2 colliers where the pneumoconiosis was probably not the cause of death. The causes of this variability are probably external variations in dust concentrations and internal variations in individual response. It also appears that the pathological classification used is too simple. It refers to the type of lesion and the maximal alteration of the lung tissue, but it does not consider the amount of lesions throughout the lung. A more elaborate classification based on type and amount of fibrosis would probably result in a better fit with the dust analyses.

The following discussion can be divided into two parts : (1) The relative hazard of different occupations, and (2) the relative hazard of different dust constituents.

Relative Hazard of Different Occupations.-A rough estimate of the relative hazard of different occupations can be obtained from the working histories and the mean pathological grading without 
reference to the lung dust analyses. The results are shown in Table 15 for those occupation groups which have a reasonable number of cases, and also for anthracite colliers and rock-workers from the old series.

TABLE 15

MEAN PATHOLOGICAL GRADE AND AVERAGE DUST EXPOSURE YEARS FOR DIFFERENT OCCUPATIONAL GROUPS

\begin{tabular}{|c|c|c|c|c|c|}
\hline Occupation & 1 & Series & No. & $\begin{array}{c}\text { Mean } \\
\text { Patho- } \\
\text { logical } \\
\text { Grade }\end{array}$ & $\underset{\text { (years) }}{\text { Exposure }}$ \\
\hline $\begin{array}{l}\text { Tin and granite } \\
\text { Rock-workers* } \\
\text { Rock-workers* } \\
\text { Colliers . } \\
\text { Colliers . } \\
\text { Hauliers, etc. }\end{array}$ & $\begin{array}{l}\cdots \\
\cdots \\
\cdots \\
\cdots\end{array}$ & $\begin{array}{l}\text { New } \\
\text { Old } \\
\text { New } \\
\text { Old } \\
\text { New } \\
\text { New }\end{array}$ & $\begin{array}{r}15 \\
5 \\
9 \\
27 \\
28 \\
10\end{array}$ & $\begin{array}{l}4 \cdot 7 \\
4 \cdot 6 \\
3 \cdot 1 \\
3 \cdot 1 \\
3 \cdot 0 \\
2 \cdot 1\end{array}$ & $\begin{array}{l}23 \\
22 \\
37 \\
39 \\
33 \\
38\end{array}$ \\
\hline
\end{tabular}

*In coal-mines.

It will be seen that the tin- and granite-workers and the rock-workers of the old series who mostly had silicosis (grade 5) after 22 and 26 years form a first group with the highest hazard.

The colliers and the rock-workers of the new series took about 10 years more (33 to 39 years) to reach grade 3 and form a second group. The hauliers, who took on an average 38 years to reach grade 2, form a third group with the smallest hazard. As Table 3 shows, these men had also been engaged for variable times as rock-workers or colliers. It is noteworthy that there is only one collier with pathological grade 1 in the series. As equal numbers of the different pathological grades were collected and as colliers are the predominating occupational group, a higher number might have been expected.

The difference between hauliers and colliers may reflect a difference in dust concentration ; this is in general higher at the coal-face than in the roadways. Hart and Aslett (1942) concluded from radiological surveys that the occupation of haulier and of other men working in the roadways had a much smaller pneumoconiosis risk than the work of the colliers. The difference between colliers and tin-miners appears to be due to a different type of disease, silicosis developing comparatively faster than pneumoconiosis of coalworkers.

The mean duration of dust exposure for different pathological grades is given in Table 16.

To what extent the figures represent a progression of disease, in the sense that it takes 26 years to reach grade 1 and another 11 or 12 years to reach grade 3 or 4, cannot be said from these data. Radiological evidence given by Fletcher (1955), however, indicates a positive relationship between simple pneumoconiosis and dosage of dust.
TABLE 16

MEAN EXPOSURE YEARS FOR DIFFERENT GROUPS

\begin{tabular}{|c|c|c|c|c|c|}
\hline \multirow{2}{*}{ Occupation } & & & \multicolumn{3}{|c|}{ Pathological Grade } \\
\hline & & 1 & 2 & 3 & 4 \\
\hline New series all cases. & . & $26(11)^{*}$ & $33(17)$ & $38(18)$ & $37(18)$ \\
\hline New series colliers only & . & - & 31 (8) & 33 (9) & $36(10)$ \\
\hline
\end{tabular}

By combining the exposure years and lung analyses one can calculate the mean amounts of dust retained per year. The results for coal, quartz, and total dust are shown in Table 17 for the main occupational groups of the new series and separately for the different pathological grades amongst the colliers. The average collier in our series retained about $1 \mathrm{~g}$. of dust per year (averaged over 33 years).

TABLE 17

MEAN RATE OF DUST ACCUMULATION FOR DIFFERENT OCCUPATIONAL GROUPS AND GRADES OF PATHOLOGY

\begin{tabular}{|c|c|c|c|c|c|c|c|c|}
\hline \multirow{2}{*}{\multicolumn{4}{|c|}{ Occupational Group }} & \multirow{2}{*}{$\begin{array}{l}\text { No. } \\
\text { of } \\
\text { Men }\end{array}$} & \multirow{2}{*}{$\begin{array}{l}\text { Patho- } \\
\text { logical } \\
\text { Grade }\end{array}$} & \multicolumn{3}{|c|}{$\begin{array}{l}\text { Dust Retained } \\
\text { per Year (mg.) }\end{array}$} \\
\hline & & & & & & $\begin{array}{l}\text { Total } \\
\text { Dust }\end{array}$ & Coal & Quartz \\
\hline $\begin{array}{l}\text { Tin-miner } \\
\text { Rock-wor } \\
\text { Hauliers } \\
\text { Colliers } \\
\text { Colliers } \\
\text { Colliers } \\
\text { Colliers }\end{array}$ & $\begin{array}{l}\ldots \\
\ldots \\
\cdots \\
\cdots\end{array}$ & $\begin{array}{l}\ddot{1-m i n e s} \\
\ldots \\
\ldots \\
\ldots \\
\ldots\end{array}$ & $\begin{array}{l}\ldots \\
\cdots \\
\cdots \\
\cdots \\
\ldots\end{array}$ & $\begin{array}{r}15 \\
9 \\
10 \\
28 \\
8 \\
9 \\
10\end{array}$ & $\begin{array}{l}\text { 二 } \\
\bar{Z} \\
2 \\
3 \\
4\end{array}$ & $\begin{array}{r}180 \\
550 \\
280 \\
1,050 \\
500 \\
1,155 \\
1,506\end{array}$ & $\begin{array}{r}80 \\
300 \\
190 \\
950 \\
435 \\
1,030 \\
1,367\end{array}$ & $\begin{array}{l}32 \\
67 \\
34 \\
27 \\
19 \\
30 \\
34\end{array}$ \\
\hline
\end{tabular}

It may be asked how much dust is needed, in terms of particles per cc. between 1 and 5 microns to yield $1 \mathrm{~g}$. of dust per year, which is the amount retained by the average collier. Assuming a breathing rate of 30 litres per minute and 300 five-hour shifts per year, the man would breathe $2,700 \mathrm{~m}^{3}$. per year, or, say $3,000 \mathrm{~m}^{3}$. while underground. Thus he would have to retain between 0.3 and $0.4 \mathrm{mg}$. of dust per $\mathrm{m}^{3}$. of air breathed. Assuming an exponential size distribution (Wynn and Dawes, 1951), 1,000 particles per cc. of size 1 to 5 microns correspond to about $5.4 \mathrm{mg}$. of dust per $\mathrm{m}^{3}$.

If all airborne dust were retained a concentration of 50 to 70 particles per cc. would give $1 \mathrm{~g}$. per year. Using the alveolar retention curve of Brown, Cook, Ney, and Hatch (1950) the dust concentration to yield $1 \mathrm{~g}$. in the lungs is approximately four times higher, leading to 200 to 300 particles per cc. of size 1 to 5 microns. This is lower than the average dust concentration found now in South Wales coal-mines and probably much lower than it had been in the days before dust suppression was practised, when the workers accumulated the dust in their lungs. But, Brown and others (1950) measured the instan- 
taneous dust retention. What is not known at present is the relation between the amount temporarily retained in the alveolar tissue and the amount permanently retained. Therefore it is not possible to estimate the average dust content of the air to which the men had been exposed from the amount of dust found in their lungs.

Relative Hazard of Different Dust Constituents.-It is generally agreed that pneumoconiosis of coalworkers is due to dust, but there are two schools of thought about the importance of quartz. In the German and American literature it is generally stated that quartz is the causative agent, and that the disease is a modified silicosis which looks different in the lungs on account of the large amounts of coal which happen to accompany the quartz. In the British literature, on the other hand, the opinion is expressed that it is the coal dust itself which mechanically by its mere presence causes the series of lung changes found in simple pneumoconiosis, but that an additional factor, possibly infective, is necessary for the initiation of P.M.F.

The fact that quartz can cause silicosis is not in doubt. Whether a completely quartz-free coal dust can cause disabling pneumoconiosis is not at present known as dusts from coal-mines are never sufficiently low in quartz to establish this point. Even coal trimmers were found to have abnormal amounts of quartz in their lungs. The best evidence could be obtained from dust exposures to carbon black or synthetic graphite if the exposures were sufficiently high and workers who worked in such dust for a sufficient length of time could be found. Gärtner and Brauss (1951) have recently examined soot-workers and found radiological abnormalities classed as grade 2 and 3 pneumoconiosis after 15 or more years' exposure to carbon black dust but they found little or no disability. This is a matter for further survey work of great importance which should be undertaken. Rüttner, Bovet, and Aufdermaur (1952) have described a case of lung fibrosis in a graphite worker, where graphite and carborundum but no quartz at all were found in the lung dust.

It is, however, not necessary to make a decision between the two theories. It appears to us possible that both excessive amounts of coal and the quartz in the dusts contribute to the disease, and our data give some information about their relative proportions. The average amounts of coal and quartz and the coal/quartz ratio for all lungs from South Wales are shown in Table 18.

The quartz in the lungs comes chiefly from rock dust and the values are useful in giving a rough idea of the relative importance of coal- and rock-
TABLE 18

RATIO OF COAL TC QUARTZ IN THE LUNGS FOR DIFFERENT GRADES OF PATHOLOGY

\begin{tabular}{|c|c|c|c|c|}
\hline \multirow{2}{*}{$\begin{array}{l}\text { Patho- } \\
\text { logical } \\
\text { Grade }\end{array}$} & \multirow{2}{*}{$\begin{array}{l}\text { No. of } \\
\text { Men in } \\
\text { Group }\end{array}$} & \multicolumn{2}{|c|}{ Dust in Lungs (g.) } & \multirow{2}{*}{$\begin{array}{l}\text { Coal/ } \\
\text { Quartz } \\
\text { Ratio }\end{array}$} \\
\hline & & Coal & Quartz & \\
\hline $\begin{array}{l}1 \\
2 \\
3 \\
4 \\
5\end{array}$ & $\begin{array}{l}12 \\
20 \\
20 \\
19 \\
15\end{array}$ & $\begin{array}{r}7.9 \\
12.4 \\
23.0 \\
40.3 \\
1.9\end{array}$ & $\begin{array}{l}0.35 \\
0.80 \\
1.19 \\
1.88 \\
1.15\end{array}$ & $\begin{array}{r}22 \cdot 6 \\
15 \cdot 5 \\
19 \cdot 3 \\
21 \cdot 4 \\
1.6\end{array}$ \\
\hline
\end{tabular}

dust, especially if the rock is rich in quartz. It is notable that the proportions of coal to quartz are approximately the same in categories 1,2 , and 3 (simple pneumoconiosis) as in category 4 (P.M.F.). This gives no support to the theory that quartz is an important factor in the aetiology of P.M.F. and agrees with Cochrane's (1955) finding that in the Rhondda Fach there was no difference in the exposure to stone dust of men with simple pneumoconiosis as compared with those with P.M.F. The findings support the view (Gough, 1947 ; Heppleston, 1951 ; James, 1954) that massive pneumoconiosis is due to a second factor, usually tuberculosis, in addition to the action of the dust. Category 4 cases are all of massive fibrosis whereas there are none of these in category 3 , yet the amount and character of the dust is not significantly different in the two groups. Thus, in these two groups, while the dust is similar, pathological changes are very different.

The action of coal and quartz on human lungs is not necessarily simply additive. Certain evidence from animal experiments suggests a more complicated mechanism. It has been shown (Ray, King, and Harrison, 1951) that very small amounts of quartz injected into the lungs of rats are eliminated from the lungs into the lymph nodes and therefore cause less lung fibrosis when they are injected alone than when they are injected with large amounts of coal which prevent this loss from the lung. From the evidence produced in this paper, however, it appears that coal may have an inhibiting effect on the fibrogenic action of quartz, possibly by simple dilution, since the amounts of quartz in the lungs of Cornish tin-miners with silicosis were very similar to those in coal-miners with simple pneumoconiosis, and the accompanying, possibly antidotal, kaolin and mica were only in slightly lower ratio to the quartz in the silicotics than in simple pneumoconiotics.

The lung dust analyses have shown that all classes of coalworkers including the colliers have more quartz in their lungs than the silicotic tinminers and granite-workers (Table 6). If the different pathological grades of colliers are separated this holds for grades 3 and 4 and only colliers of 
grade 2 have slightly less. It is true for the total quartz in the lungs and for the calculated annual increments shown in Table 17. But we cannot say from our findings whether the coal, kaolin, or mica is inhibiting the effect of the quartz or whether they are themselves responsible for the fibrosis and could have caused it in the absence of quartz.

\section{Summary}

In continuation of previous work lung dust analyses were made of 71 pneumoconiotic coalworkers from South Wales and of 15 silicotic tinminers and granite-workers from Cornwall. In classifying the material five pathological grades and seven occupational groups were distinguished.

Coal, total silica, quartz, and the sum of kaolin and mica were determined by chemical and $x$-ray diffraction methods and the amounts of these constituents and of the total dust in the lungs were estimated.

The dust composition was found to vary for different occupational groups. The chief characteristic was the percentage of coal in the dust which fell in going from colliers to hauliers, rock-workers, and tin- and granite-workers. The quartz percentage of the siliceous minerals rose slightly in the same order of occupations. "Coal" in tin-miners" lungs is probably chiefly soot from acetylene lamps or candles and atmospheric pollution.

The amount of dust in the lungs increased with the pathological grade but differences between grades 3 and 4 were not significant. This lends support to the view that a second factor is necessary to account for the difference. On an average the colliers' lungs contained $35 \mathrm{~g}$. of total dust of which $0.9 \mathrm{~g}$. was quartz. The tin-miners had $4 \mathrm{~g}$. of total dust and $0.7 \mathrm{~g}$. of quartz. The calculated average dust accumulation rate for colliers was of the order of $1 \mathrm{~g}$. of dust per year, but it is doubtful whether this rate is constant.

Although the quartz percentage of the lung dust was much higher in silicotic tin-miners than in colliers with pneumoconiosis, the absolute amounts of quartz in their lungs were similar. Rock-workers in coal-mines had over three times more quartz in their lungs than the silicotic tin-miners.

The proportion of coal to quartz for the whole group of lungs from South Wales was of the order of $20: 1$. It appears likely that both total dust and quartz contribute to the pneumoconiosis of coalworkers.

We are grateful to Professor J. Gough who provided the lungs from South Wales, made the pathological assessments, and secured the working histories, and to Dr. L. W. Hale, who provided the lungs from Cornwall and their working histories. A detailed pathological description of the material will be published by Professor J. Gough. Miss V. Pash and Mr. W. Weeden gave help with the analyses and Miss A. V. Sanderson and Mrs. C. Wrightam with the computations. We are also grateful to Dr. J. C. Gilson and the staff of the Pneumoconiosis Research Unit for $x$-ray readings and helpful criticism, to Dr. J. O. Irwin for statistical advice, and to the Medical Research Council for financial assistance. Most of the $x$-ray diffraction analyses were made at the National Physical Laboratory. Figs. 6 to 10 are reproduced by permission of the Controller, H.M. Stationery Office.

\section{REFERENCES}

Badham, C., and Taylor, H. B. (1938). Report of the DirectorGeneral of Public Health, New South Wales, 1936, p. 100

Blacklock, J. W. S., Kennaway, E. L., Lewis, G. M., and Urquhart, M.'E. (1954). Brit. J. Cancer, 8, 40

Brown, J. H., Cook, K. M. Ney, F. G., and Hatch, T. (1950), Amer. J. Publ. Hlth, 40, 450 .

Cartwright, J., and Nagelschmidt, G. (1951). S.M.R.E. Res. Rep. No. 24

Cochrane, A. L. (1954). Brit. J. Tuberc., 48, 274.

(1955). Personal communication.

Davies, I., and Fletcher, C. M. (1951). British Journal of Industrial Medicine, 8, 244.

Fletcher, C. M. (1955). Arch. industr. Hlth, 11, 29.

Gärtner, H., and Brauss, F. W. (1951). Med. Welt, 20, 253.

Gentry, C. H. R., and Sherrington, L. G. (1946). Analyst, 71, 432.

Gough, J. (1947). Occup. Med., 4, 86.

Gough, J. (1947). Occup. Med., 4, 86.

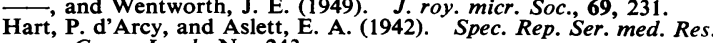
Coun., Lond., No. 243.

Heppleston, A. G. (1951). Arch. industr. Hyg., 4, 270.

James, W. R. L. (1954). Brit. J. Tuberc., 48, 89.

King, E. J. (1939). Biochem. J., 33, 944. and Gilchrist, M. (1945). Spec. Rep. Ser. med. Res. Coun.,

, and Nagelschmidt, G. (1945). Ibid., No. 250B.

Policard, A., and Collet, A. (1953). Arch. Mal. prof., 14, 337.

Ray, S. C., King, E. J., and Harrison, C. V. (1951). British Journal of Industrial Medicine, 8, 62 .

Rüttner, J. R., Bovet, P., and Aufdermaur, M. (1952). Dtsch. med. Wschr., 77,1413 .

Stacy, B. D., and King, E. J. (1954). British Jounral of Industrial Medicine, 11, 192.

Wynn, A. H. A., and Dawes, J. G. (1951). S.M.R.E. Res. Rep. No. 28. 